\title{
Load optimization for reducing the cost of an electric vehicle's battery pack
}

\author{
Bruno Cárdenas*, Seamus D. Garvey \\ Department of Mechanical, Materials and Manufacturing Engineering, University of Nottingham, \\ University Park, NG7 2RD, United Kingdom
}

\begin{abstract}
This paper presents the cost-optimization of the $24 \mathrm{kWh}$ battery pack of a Nissan Leaf. The optimization is based on decomposing the load that the battery experiences into two frequency components, which are handled by two independent batteries. The reduction in cost comes from the possibility of manufacturing batteries of different specifications whose cost per unit energy $(\$ / \mathrm{kWh})$ and per unit power $(\$ / \mathrm{kW})$ differ considerably from each other. The High-Capacity battery used for the low frequency part of the load has a low cost per unit energy capacity and a higher cost per unit power whilst the High-Power battery used for the fast-frequency part of the load is the reverse case.

Two case studies were carried out. The first one uses the load profile seen by the battery pack when the car is subjected to the EPA-LA92 driving cycle. The second case study considers a modified profile with a much higher crest factor. A sign-preserving filter is used in the study to perform the signal splitting. A two-dimensional search space is created and numerous "splits" are explored.

Results show that an important reduction in the cost of the battery can be achieved. In the optimum configuration found (for the high crest factor profile) the High-Capacity battery has a capacity of $19.23 \mathrm{kWh}$ and sees a peak power of $37.17 \mathrm{~kW}$; whereas the High-Power battery has a capacity of $4.77 \mathrm{kWh}$ but sees a much larger peak power of $88.56 \mathrm{~kW}$. The total cost of this hybrid system is $\$ 5939$, which represents a $12.7 \%$ cost reduction with respect to the original battery of the vehicle.
\end{abstract}

\section{Keywords}

Battery cost reduction; load optimization; signal splitting; hybrid system; electric vehicle; sign-preserving filter

\section{Introduction}

The transportation sector is of paramount importance for the wellbeing of the population and for promoting economic growth. Over the past 30 years the world's energy use for transport has more than doubled [1,2] and in 2017 it accounted for approximately $30 \%$ of the world's total energy consumption [3], from which $44 \%$ is attributable to light-duty cars and trucks [4].

It is a well-known fact that the number of vehicles transiting on public roads has seen a dramatic increase in recent years. Approximately 393 million new vehicles have been sold worldwide between 1990 and 2017; of which 76\% were sold during the past 4 years [5]. Forecasts suggest that sales will keep on the rise and an increase of $3.6 \%$ is projected for 2018 [6].

The exponential growth of the transportation sector has caused, among other problems, an increase in harmful greenhouse gas emissions, particularly $\mathrm{CO}_{2}$. It is estimated that the transportation sector accounts for $\sim 7.5$ billion metric tons per year of $\mathrm{CO}_{2}$ emissions, which is equivalent to a $23 \%$ of the world's total. Nearly $80 \%$ of the emissions of the transportation sector are attributable to light-duty vehicles [7, 8].

The world has recognized the severe environmental impact of excessive and sustained carbon dioxide emissions and a global effort has been undertaken to shift towards a carbon neutral economy. The energy sector has seen a rise in renewable generation; nevertheless considerable improvements in the transportation sector are also needed since it is one of the economy's largest sources of greenhouse gases.
Electric vehicles (EVs) are an innovative zero-emission technology with the potential of playing a major role in the world's transition towards a sustainable economy. Governments of numerous countries have created action plans, installed regulatory instruments and dedicated considerable amounts of funding to promote the development and adoption of EVs, as a measure not only to reduce carbon dioxide emissions but to diminish the global dependency to oil as well.

There is undoubtedly a lot of momentum around EVs, which have also attracted significant interest and investment from the private sector. Since 2010, more than 20 automakers have introduced EVs to their line-ups [9] and in 2016 global electric vehicle ownership surpassed the 2 million mark [10]. The market share of EVs will continue to grow; nevertheless the forecast growth rate is not rapid enough. Despite changes made in legislations, better efficiencies achieved by all transport technologies and an increasing number of EVs on the road, emissions from the transportation sector are expected to increase an astounding $60 \%$ by 2050 [11].

There are still several challenges and issues to address in order to achieve a much more widespread deployment and utilization of EVs. These range from vehicle-related aspects such as high cost and short life, battery technology limitations and inaccurate real-world driving range estimations to infrastructure-related aspects such as the capability of the electric grid to handle an increased demand of power throughout the day and the location and amount of public charging stations. A substantial amount of research has been devoted globally to find solutions to the aforementioned barriers; notwithstanding, considerable effort is still needed to promote the widespread utilization of EVs. 


\subsection{Objective of the study}

Accordingly, in this paper a study on the cost-optimization of the battery-pack of an EV is presented. The study has two main objectives: (i) demonstrate the use of a signal processing tool (namely a "Sign-Preserving Filter") in an EV context and (ii) understand the potential reduction in the cost of the battery pack (hence overall cost of the EV) if said battery is replaced by a hybrid 2-battery system, where one battery is used for bulk energy storage and the other battery is used for supplying power peaks.

An energy consumption model is used to create the power profile seen by a Nissan Leaf when subjected to a standard driving cycle "EPA-LA92". This power profile is assumed to be representative of the driving conditions an EV is most likely to experience and is used as a case study. A "Sign-Preserving Filter" is used to split the power profile into two independent signals, each of which will be handled by one of the batteries of the hybrid system.

A combinatorial space is created by varying the two control parameters of the filter, which in effect creates an array of different ways in which the power profile can be split. A cost model is used to evaluate the behaviour of the total cost of the system with respect to the control parameters and identify the optimum combination from an economic point of view.

\section{Models and Tools used for the Optimization}

\subsection{Driving Schedule}

In the automotive industry numerous tests are carried out on vehicles to assess different parameters such as exhaust emissions, range and component durability. The vehicles are tested using a chassis dynamometer on which they perform a pre-defined driving cycle and experience simulated frictional losses and aerodynamic resistance. Driving cycles are usually defined in terms of vehicle speed and gear selection as a function of time [12].

In the present study the California LA92 Driving Schedule of the Environmental Protection Agency (EPA) is used. This cycle was selected because it is a transient cycle (speed and acceleration are changing continuously) that accurately reflects driving conditions in an urban environment, which is the type of situation that electric vehicles are most likely to encounter $[13,14]$.

The "EPA LA92" driving schedule, shown in Figure 1, has a duration of $1435 \mathrm{~s}$ in which $15.8 \mathrm{~km}$ are covered. The top speed reached is $108.1 \mathrm{~km} / \mathrm{hr}$ and an average of $39.6 \mathrm{~km} / \mathrm{hr}$ is observed. The cycle considers 233 seconds out of the 1435 at full stop (i.e. speed $=0$ ). The maximum positive acceleration during the cycle is $3.1 \mathrm{~m} / \mathrm{s}^{2}$ while the maximum deceleration is $3.9 \mathrm{~m} / \mathrm{s}^{2}$. The second-to-second dataset comprising the LA92 driving cycle can be downloaded from the EPA's website [15].
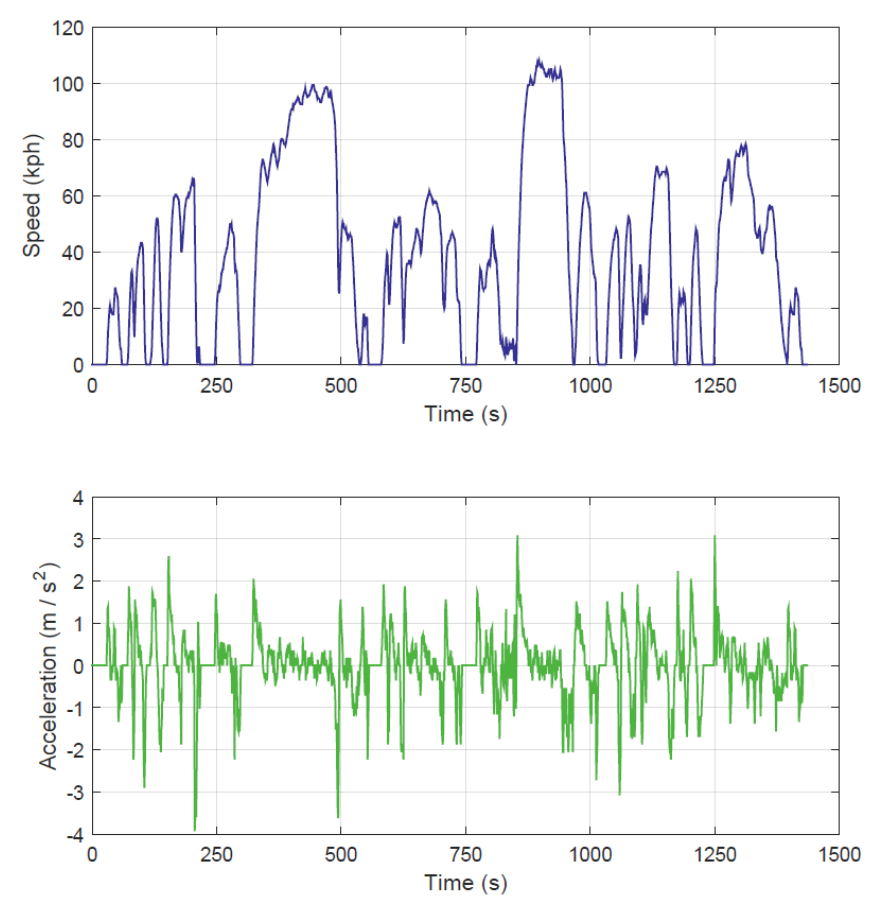

Figure 1. Speed and acceleration profiles of the EPA California LA92 driving schedule.

\subsection{Vehicle Energy Consumption Model}

In order to carry out the optimization of the duty of the battery, the driving cycle needs to be translated into an electric load. The speed-to-electric power conversion is carried out by means of the power-based vehicle energy consumption model (VTCPEM) developed by Virginia Tech. [16].

The model computes the instantaneous energy consumption of an EV using second-by-second vehicle speed, acceleration and roadway grade data as input variables. Additionally, it computes the regenerative braking efficiency using the instantaneous vehicle operation variables in contrast to other models that use average constant regenerative braking efficiency [17-19].

The VT-CPEM is a general model that can be applied to any electric vehicle. The model was validated by the authors with experimental data from a Nissan Leaf [20, 21]. For this study the same reference vehicle is used.

The model calculates the power at the wheels $\left(P_{w}(t)\right)$ by means of Eq. (1) using as inputs the speed of the vehicle (given by the driving schedule), its physical characteristics and the road's conditions [16].

$$
P_{w}(t)=\left[m a(t)+F_{1}+F_{2}+m g \cdot \sin (\theta)\right] \cdot v(t)
$$

Where:

$$
F_{1}=m g \cdot \cos (\theta) \cdot \frac{C_{r}}{1000} \cdot\left(C_{1} v(t)+C_{2}\right)
$$




$$
F_{2}=\frac{1}{2} \cdot \rho \cdot A_{f} \cdot C_{D} \cdot v^{2}(t)
$$

In Equations (1)-(3) $m$ is the mass of the vehicle, $v(t)$ is the velocity (given by the driving schedule), $a(t)$ is the acceleration (which takes negative values when the vehicle brakes), $g$ is the gravitational acceleration and $\theta$ is the road grade (or slope). The coefficients $C_{r}, C_{l}$ and $C_{2}$ are rolling resistance parameters that vary as a function of road surface type, road condition and vehicle tire type, respectively. The density of the air is represented by $\rho, A_{f}$ is the frontal area of the car and $C_{D}$ is the aerodynamic drag coefficient of the vehicle.

The model calculates the power at the electric motor $\left(P_{e}(t)\right)$ based on the power seen at the wheels, by means of Eq. (4), which takes into consideration the efficiency of the drivetrain $\left(\eta_{D}\right)$ and the efficiency of the electric motor itself $\left(\eta_{e}\right)$ [16]. The parameters used in the study to calculate the electric power at the wheels are given in Table 1.

$$
P_{e}(t)=\left\{\begin{array}{cc}
\frac{P_{w}}{\eta_{D} \cdot \eta_{e}} & P_{w}>0 \\
0 & P_{w}=0 \\
\frac{P_{w}}{\eta_{D} \cdot \eta_{e}} \cdot \eta_{r b} & P_{w}<0
\end{array}\right.
$$

Where:

$$
\eta_{r b}=\exp \left(\frac{0.0411}{a(t)}\right)
$$

With the values listed in Table 1, the EPA-LA92 drive cycle translates into the power profile shown by Figure 2. The power profile shown in the figure is specific of a Nissan Leaf; a different vehicle subjected to the same driving cycle would exhibit a different power profile.

Table 1. Input parameters for the VT-CPEM model.

\begin{tabular}{cccc}
\hline Parameter & Value & Units & Reference \\
\hline$m$ & 1521 & $\mathrm{~kg}$ & {$[22]$} \\
$g$ & 9.81 & $\mathrm{~m} / \mathrm{s}^{2}$ & -- \\
$\theta$ & 0 & $\circ$ & -- \\
$C_{r}$ & 1.75 & -- & {$[23]$} \\
$C_{I}$ & 0.033 & -- & {$[23]$} \\
$C_{2}$ & 4.575 & -- & {$[23]$} \\
$\rho$ & 1.226 & $\mathrm{~kg}^{3}$ & -- \\
$A_{f}$ & 2.332 & $\mathrm{~m}^{2}$ & {$[16]$} \\
$C_{D}$ & 0.28 & -- & {$[22]$} \\
$\eta_{D}$ & 0.92 & -- & {$[24]$} \\
$\eta_{e}$ & 0.91 & -- & {$[16]$} \\
\hline
\end{tabular}

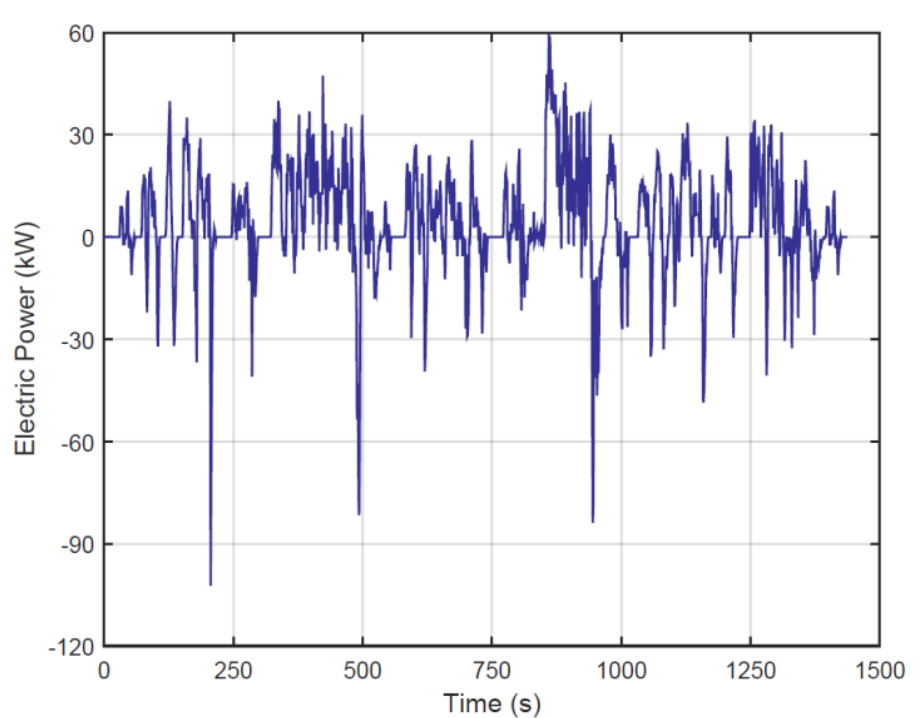

Figure 2. Electric power profile of a Nissan Leaf when subjected to the EPA-LA92 drive cycle.

\subsection{The Signal Splitting Tool}

The objective of this study is, as aforementioned, to explore the possibility of achieving a reduction in the cost of the electric battery of an EV. The premise is that the energy store could be hybridized in the following way: The duty (profile illustrated in Fig. 2) can be split into a predominantly low frequency profile and a predominantly high frequency profile. The lowfrequency profile will have a large energy content and a reduced peak power whereas the high-frequency profile will have a small energy content but a comparatively large peak power.

Instead of having a single battery servicing the load in its entirety, the EV will have 2 batteries, one for each of the two frequency components (low and high) of the load. The two batteries will be based on different compositions/technologies and therefore their cost will be different. The low-frequency battery will have a much lower cost per unit of energy storage capacity $(\$ / k W h)$ while it will have a high cost per unit power $(\$ / k W)$. Conversely, the high-frequency battery will have a much lower cost per unit power but will have an increased cost per unit energy. Therefore, the low-frequency battery will supply the bulk of the energy and the high-frequency battery will mainly function as a peak-shaver. The low and high frequency batteries will be hereafter referred to as "High Capacity" and "High Power", respectively.

The intention of this paper is to demonstrate the use of a signal splitting tool (namely the sign preserving filter) for the cost optimization of the battery pack in an EV. The optimization presupposes the possibility of manufacturing batteries of different specifications whose cost per unit energy and per unit power differ considerably from each other. The selection of the two specific types of technologies for the High-Capacity and High-Power batteries is out of the scope of the paper. Nonetheless, section 3.1 presents a qualitative discussion on different types of battery chemistries together with examples of possible combinations thereof. 
The most straightforward way to divide a signal (electric load in this case) is to employ a traditional signal-processing method, such as a Fourier analysis, to obtain the different frequency components and group them into a low frequency signal and a high frequency signal. However, such methods are not appropriate for an EV application for one reason: counterflow of energy. Figure 3 shows an example of two signals obtained after decomposing the EPA-LA92 power profile via a Fourier analysis.

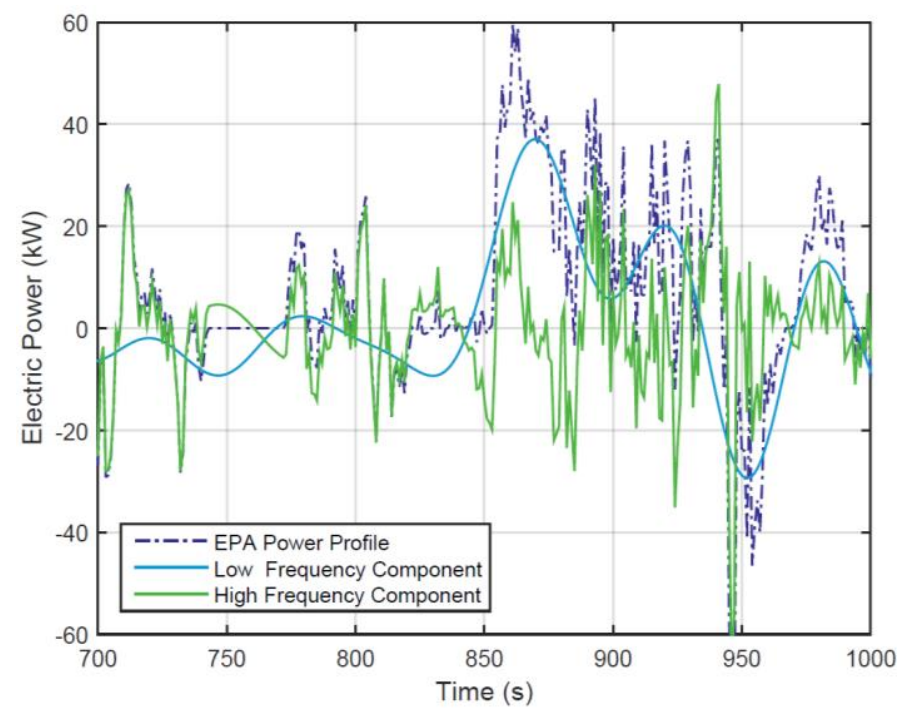

Figure 3. Example of a Fourier decomposition (and grouping) of the power profile

It can be seen that in many instants one signal is positive while the other is negative. In the context of a car this implies that one battery is being charged by the regenerative brakes while at the same time the other battery is in discharge (power flowing to the wheels). Needless to say, the situation described does not make sense; both batteries should be either charging or discharging at the same time. The case where one battery is idle while the other one is active is possible because there is no work in opposite directions.

A "Sign-Preserving Filter" capable of splitting a signal $\boldsymbol{A}$ into two components $\boldsymbol{B}$ and $\boldsymbol{C}$ in such a way that at every point in time $(t)$ the two components have the same sign as $\boldsymbol{A}$ and their sum equals $\boldsymbol{A}$ has been proposed by Cárdenas et al. [25]

$$
\begin{aligned}
& A(t)=B(t)+C(t) \\
& \operatorname{sgn}[A(t)]=\operatorname{sgn}[B(t)]=\operatorname{sgn}[C(t)]
\end{aligned}
$$

The main difference between the sign-preserving filter and other Fourier-based filters is that the condition described by Eq. (7) is true at all times. During the periods when the original signal $\boldsymbol{A}$ is positive, $\boldsymbol{B}$ and $\boldsymbol{C}$ are also positive. Similarly, during the periods when $\boldsymbol{A}$ is negative, both components have a negative value as well. The component $\boldsymbol{B}$ produced by the filter contains mainly low-frequency (smooth) content while $\boldsymbol{C}$ is predominantly comprised of high frequency (not-so-smooth) content.
The aforementioned sign-preserving filter is used as a tool in this study for the cost-optimization of the battery pack of an EV. A brief explanation of the operation of the filter is provided but its particularities are not addressed in depth. A comprehensive explanation of the mechanics of the filter, together with examples of other applications, can be found in [25].

The operation of the filter is based on wavelets. The inputs to the filter are a discrete signal (in this case the electric power profile of a Nissan Leaf when subjected to the EPA-LA92 driving cycle) and two user-defined parameters: maximum width of the wavelets $\left(\omega_{\max }\right)$ and number of runs $\left(n_{\text {run }}\right)$.

Figure 4 shows the process followed by the filter. In every one run the filter generates co-sinusoidal wavelets of $3,5,7 \ldots \omega_{\max }$ number of points. An important consideration is that the wavelets should be relatively small compared to the length (total number of points) of the signal.

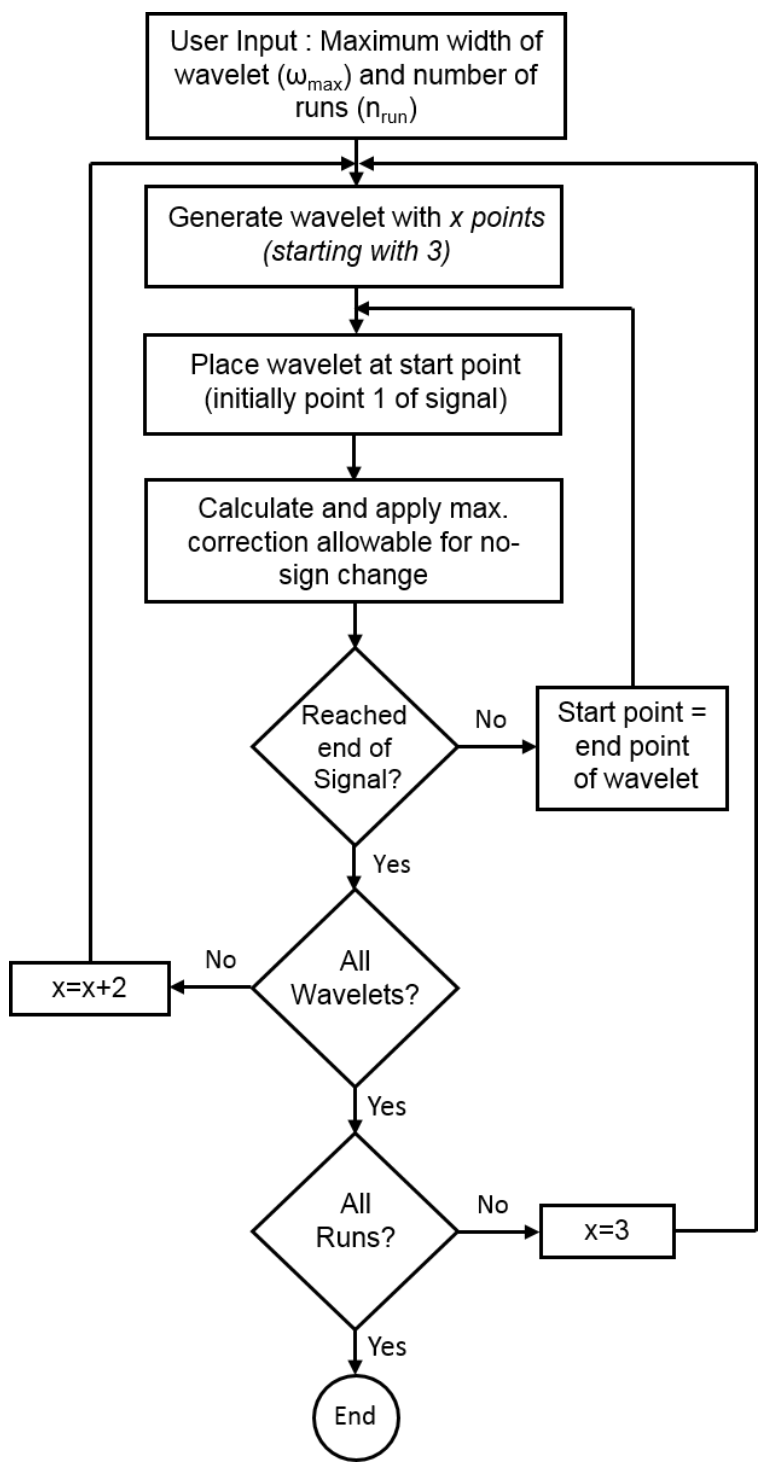

Figure 4. Simplified flowchart of the operation of the SignPreserving Filter 
The low-frequency component $\boldsymbol{B}$ starts the process being an identical copy of the original signal $\boldsymbol{A}$ while the high-frequency signal $\boldsymbol{C}$ starts being completely zero. Every wavelet marches through the signal, the smallest one ( 3 points) is the first while the widest one $\left(\omega_{\max }\right)$ is the last one. As a wavelet moves through the signal it defines an "interval of interest".

Within that "interval of interest" the filter calculates (based on the shape of the wavelet) the maximum amount of nonsmoothness (i.e. high frequency) that it can remove from the $\boldsymbol{B}$ component and add to the $\boldsymbol{C}$ component without causing a sign change in either of them.

Once a wavelet has passed throughout the complete signal the process is repeated with the following wavelet. One run of the filter is completed when all the wavelets comprised in the interval between 3 points and $\omega_{\max }$ have passed through the signal. The filter can run as many times as the user defines via $n_{\text {run }}$.

As expected, a larger $n_{\text {run }}$ yields a smoother low-frequency signal. However, the maximum wavelet width has a stronger effect on the performance of the filter. When a small value for $\omega_{\max }$ is used the filter can only remove a very small amount of "non-smoothness" on every run because it does fewer passes per run (proportional to the number of wavelets). Conversely, when a larger value for $\omega_{\max }$ is considered, more wavelets are used per run therefore the filter is able to subtract larger amounts of high-frequency in every run and produces a much smoother curve in less iterations.

Figure 5 shows an example of a split of the EPA- LA92 power profile done with the sign-preserving filter using a $\omega_{\max }$ of 25 and 50 runs. The figure shows only a small segment of the profile (between 570 and 750 seconds) for the sake of an easier visualization. It can be seen that the conditions stated by Eqs.(6)-(7) are effectively met at all times.

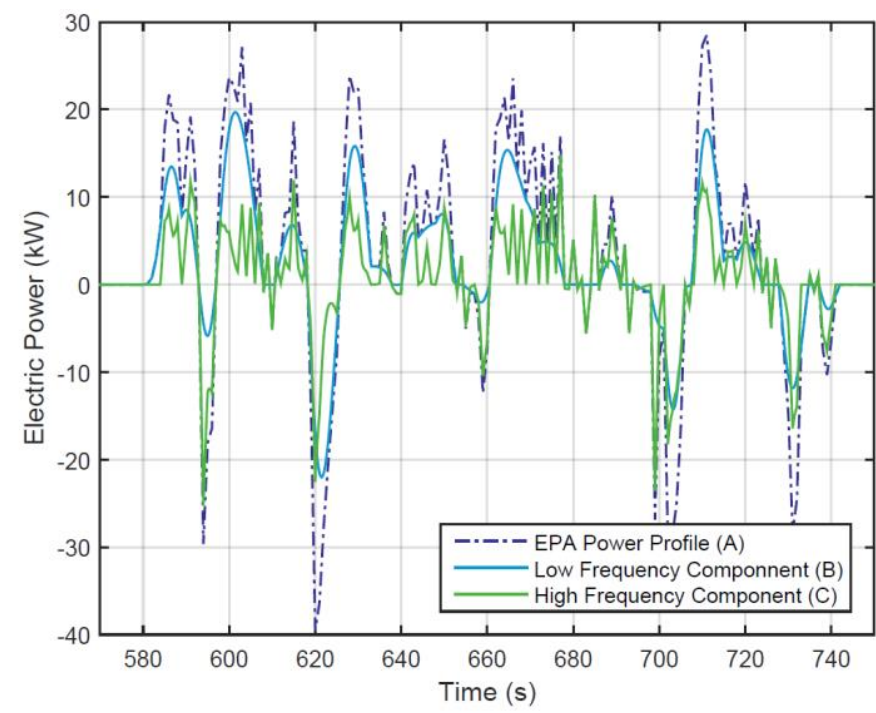

Figure 5. Example of a signal split produced by the sign-preserving filter
Another particularity of the Sign-Preserving filter that should be pointed out is the fact that the filter does not have a stopping condition other than the specified number of runs. As aforementioned, the signal $\boldsymbol{B}$ is initialized as a copy of the original signal $\boldsymbol{A}$ and the signal $\boldsymbol{C}$ starts the process being a flat line at zero. The filter removes "high frequency" content from signal $\boldsymbol{B}$ and adds it to signal $\boldsymbol{C}$. If $n_{\text {run }}$ is sufficiently large, the signal $\boldsymbol{B}$ will end up being completely smoothed down to zero and signal $\boldsymbol{C}$ will end up being an identical copy of the original signal $\boldsymbol{A}$. The key point is that the operation of the filter is a selective process: the highest-frequencies found in each pass are removed first and the lower frequencies are left behind for a subsequent pass.

\section{Analysis of Results and Discussion}

\subsection{Case study 1: Nissan Leaf under EPA-LA92}

The use of the sign preserving filter will be demonstrated through the optimization of a Nissan Leaf's battery pack. It must be highlighted that the optimization technique presented in this paper is not limited to cars, but could be equally applied to other types of EVs, being particularly beneficial to vehicles that are likely to experience drastic or very frequent acceleration and braking, such as electric bikes and city buses.

The signal used for the case study no. 1 is the electric power profile of the vehicle (see Fig. 2) when it is subjected to the EPA-LA92 driving cycle. The signal has a maximum peak power of $102.35 \mathrm{~kW}$ and an average (RMS) power of 16.22 $\mathrm{kW}$. With those two values a crest factor with a magnitude of 6.31 is calculated via Eq. (8). The crest factor is a measure of how extreme peaks are in a given signal; the closer that number is to one the closer the signal is to a straight line. At the end of the drive cycle the net energy outflow from the battery is $1.84 \mathrm{kWh}$; throughout the cycle the battery supplies the $3.07 \mathrm{kWh}$ to the electric motor but $1.23 \mathrm{kWh}$ are recuperated thanks to the regenerative brakes of the vehicle.

$$
\operatorname{Crest}(x)=\frac{\left|x_{\text {peak }}\right|}{x_{R M S}}
$$

The Nissan Leaf has a total energy storage capacity of $24 \mathrm{kWh}$ provided by a single Li-ion battery pack (comprised of 192 cells) [26]. In this study, the total storage capacity of the vehicle is treated as a constraint and is maintained constant at $24 \mathrm{kWh}$, which is supplied by the combined capacities of the High-Capacity and High-Power batteries.

The original battery of the Nissan Leaf has a Peak Power to Energy Capacity ratio (PPtE) of 4.26 (considering the EPALA92 profile). In the cost-optimization of the battery pack the PPtE for the High-Capacity battery needs to be minimized while the PPtE of the High-Power battery needs to be maximized. That is to say, the High-Capacity battery will have a large storage capacity (most of the $24 \mathrm{kWh}$ ) and will handle a reduced (or steady) power demand while the High-Power battery will be much smaller in capacity but will experience comparatively large power peaks. 
There is a wide range of battery chemistries, each with different advantages and drawbacks. Traditional batteries such as Lead and $\mathrm{Ni}-\mathrm{MH}$, although still used for numerous applications, are not used in EVs as they are considered as mature technologies that have reached their peak in cost reduction and performance. Li-ion is regarded as the most promising battery technology for the near future due to its favorable characteristics: high energy density, high efficiency, long lifespan. Among the most relevant commercially available $\mathrm{Li}$-ion batteries we can find the following chemistries:

- $\quad$ Lithium-Cobalt Oxide $\left(\mathrm{LiCoO}_{2}\right)$

- Lithium Manganese-Oxide $\left(\mathrm{LiMn}_{2} \mathrm{O}_{4}\right)$,

- Lithium Nickel Manganese Cobalt-Oxide $\left(\mathrm{LiNiMnCoO}_{2}\right)$

- Lithium Iron-Phosphate $\left(\mathrm{LiFePO}_{4}\right)$

- Lithium Nickel Cobalt Aluminium-Oxide $\left(\mathrm{LiNiCoAlO}_{2}\right)$

- Lithium Titanate $\left(\mathrm{Li}_{4} \mathrm{Ti}_{5} \mathrm{O}_{12}\right)$

Figure 6 shows a graphic summary of the main characteristics of said commercially available batteries [27]. It can be easily seen that there are trade-offs between the different characteristics of the batteries; generally one parameter cannot be improved without worsening another.

The Nissan Leaf uses a battery pack based on Lithium Manganese-Oxide (commonly known as LMO) due to a reasonable good specific power. However the specific energy of the composition is somewhat compromised, which ultimately results in a reduced driving range. Tesla, on the other hand, employs batteries based on Lithium-Nickel-Cobalt Aluminum-Oxide (commonly known as NCA) for its EVs. The NCA composition has clear advantages over LMO; nevertheless it compromises power in favor of energy.

The Lithium-Nickel-Manganese Cobalt-Oxide (NMC) batteries offer the best balance among all commercial solutions. A remarkable feature of this composition is that the proportions can be varied to modify the characteristics of the battery. Increasing the share of nickel favours the specific energy while increasing the share of manganese increases the specific power. The NMC batteries dominate the electric vehicle market [28].

There are other battery chemistries not based on lithium such as the Sodium Nickel-Chloride $\left(\mathrm{Na} / \mathrm{NiCl}_{2}\right)$ composition. Batteries based on this chemistry can achieve a cost of about one third of the cost of a Li-ion battery while having a comparable specific energy, they have a long lifecycle and can be discharged almost completely without suffering any degradation. However their specific power is much lower than Li-ion; hence they haven't been considered as the main battery for EVs [29].

The High-Capacity battery of the proposed 2-battery hybrid system could be based, for example, on a chemistry that has a high specific energy such as NCA, NMC or even $\mathrm{Na} / \mathrm{NiCl}_{2}$. The HP battery, on the other hand, could be based on a chemistry with a high specific power such as LFP.
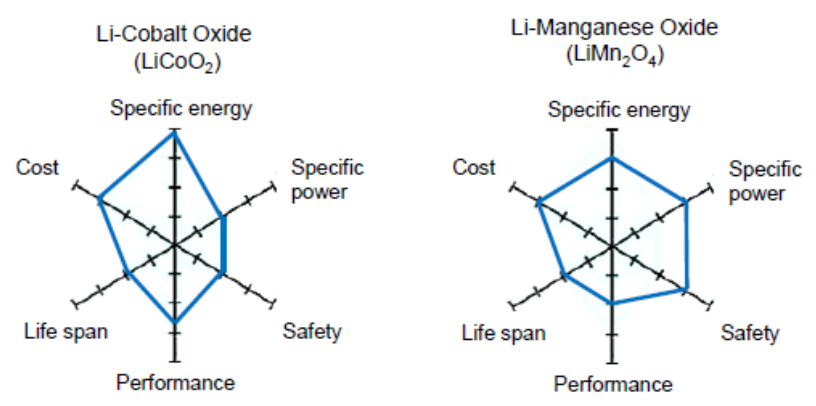

Lithium Nickel Manganese Cobalt Oxide
\[ \left(\mathrm{LiNiMnCoO}_{2}\right) \]

Lithium Nickel Cobalt Aluminium Oxide

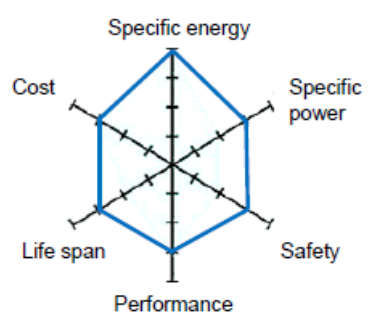
$\left.(\mathrm{LiNiCoAlO})_{2}\right)$
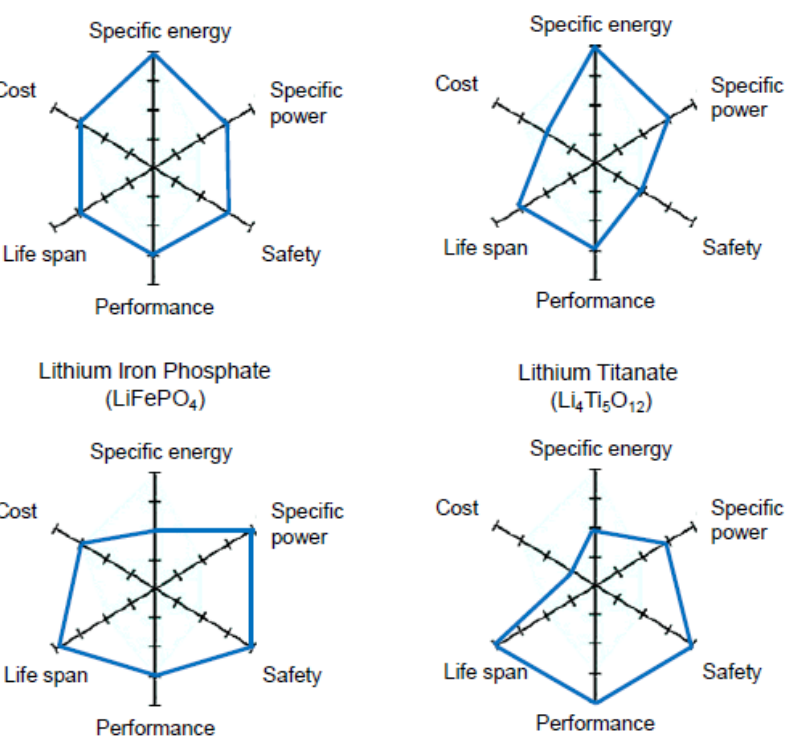

Figure 6. Summary of characteristics of commercial Li-ion chemistries

It is important to highlight that the aforementioned combination of chemistries (NMC + LFP) is merely an example. As mentioned in section 2.3, the paper is not aimed at designing a specific hybrid battery pack; instead its intention is to demonstrate the use of the sign preserving filter for optimizing (in terms of cost) the battery pack of an EV. It could very well be the case that the tool is used in future years when new battery compositions that are currently under development become available.

To carry out the cost optimization of the battery-pack of the Nissan Leaf, a 2D combinatorial space is created with different values for the two input parameters to the filter: $\omega_{\max }$ and $n_{\text {run }}$. The maximum wave width spans a range from 3 to 71 points (excluding even values) while the number of runs goes from 1 up to 100. In other words, 3500 different filtering operations will be carried out with the EPA-LA92 power profile, which will produce 3500 different load profiles for each one of the batteries (High-Capacity and High-Power). The ranges for $\omega_{\max }$ and $n_{\text {run }}$ may seem to be rather arbitrarily defined; however Figure 10 demonstrates that the optimum solution (least cost) is contained within the defined 2D search space. 
A capacity ratio $\left(E_{H C}\right)$ defined as the net amount of energy supplied by the High-Capacity battery with respect to net energy supplied by the original battery is calculated, through Eq. (9), for all of the 3500 different signal splits generated. The High-Power battery provides the difference in capacity between the original battery $(24 \mathrm{kWh})$ and the capacity of the High-Capacity battery. Therefore its capacity ratio $\left(E_{H P}\right)$ is simply 1 minus the capacity ratio of the High-Capacity battery.

$$
\begin{aligned}
E_{H C} & =\int_{t=0}^{t=1435} B(t) d t / \int_{t=0}^{t=1435} A(t) d t \\
E_{H P} & =1-E_{H C}
\end{aligned}
$$

Figure 7 shows how the capacity ratio of the High-Capacity battery $\left(E_{H C}\right)$ varies with respect to the filter's control parameters. It can be seen that the capacity ratio is high (> 95\%) for small values of $\omega_{\max }$ and small values of $n_{\text {run }}$, which indicates that the High-Capacity battery is supplying almost all of the energy required by the driving schedule. This occurs because when the filter operates with a small maximum wave-width $\left(\omega_{\max }\right)$ it is only capable of removing a very small amount of non-smoothness per run; therefore signal $\boldsymbol{B}$ (low frequency) ends up being very similar to the original signal $\boldsymbol{A}$. Conversely, for large values of $\omega_{\max }$ and large values of $n_{\text {run }}$ the capacity ratio $\left(E_{H C}\right)$ is very low $(<0.3)$, which indicates that the High-Capacity battery is doing very little and the majority of the work is being done by the High-Power battery.

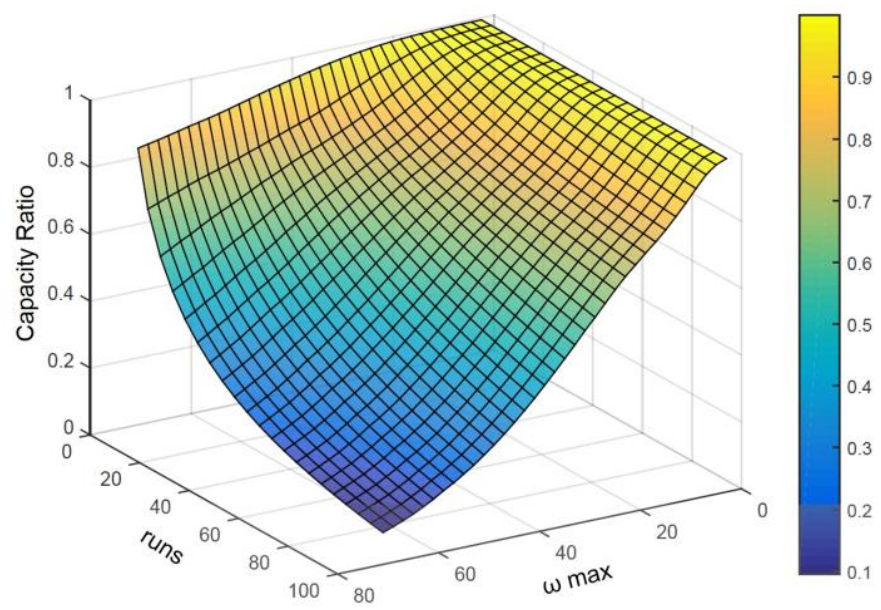

Figure 7. Variation of the capacity ratio of the High-Capacity battery $\left(E_{H C}\right)$ with respect to the filter's control parameters

Figure 8 shows the peak power demand experienced by both batteries with respect to the control parameters of the filter. It can be observed that at low values of $\omega_{\max }$ and $n_{\text {run }}$ the peak power seen by the High-Capacity battery is very close to the peak power of the original unfiltered EPA-LA92 signal $(102.35 \mathrm{~kW})$ and that the peak power handled by the HighPower battery is very low. The foregoing is caused by the filter's inability to remove significant amounts of nonsmoothness per run when it operates with small values of $\omega_{\max }$.
At high values of $\omega_{\max }$ and $n_{\text {run }}$ the inverse case occurs. The peak power experienced by the High-Capacity battery reduces dramatically while the peak values seen by the High-Power battery approximate the absolute maximum peak power of the unfiltered EPA-LA92 signal.

It is very important to emphasize that even though the sum of the low-frequency $(\boldsymbol{B})$ and fast-frequency $(\boldsymbol{C})$ signals replicate exactly the original signal $(\boldsymbol{A})$, the sum of the peak powers of $\boldsymbol{B}$ and $\boldsymbol{C}$ might exceed the peak power of $\boldsymbol{A}$ because they might not occur at the same instant in time.

The peak power plots (Fig.8) reveal important information about how the sign-preserving filter is acting. The objective of the optimization process is in effect to reduce the power requirement for the High-Capacity battery in order to reduce its cost and shift those power peaks to a more suitable HighPower battery. Nevertheless; these results can be misleading and conclusions should not be drawn exclusively from this point of view.

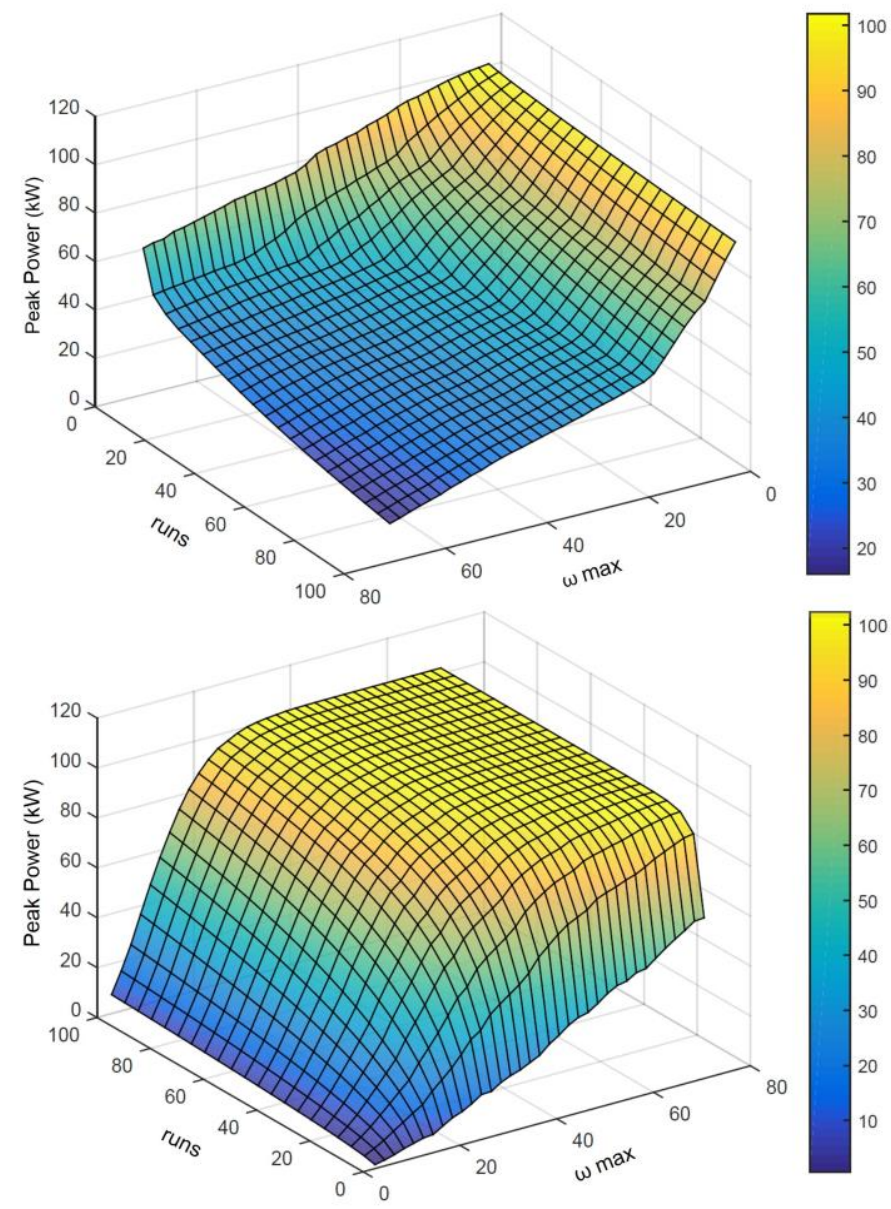

Figure 8. Variation of the peak power seen by the High-Capacity (top) and High-Power (bottom) batteries with respect to the filter's control parameters

In the figures, the portion of the surface where the HighCapacity battery experiences a small peak power (large $\omega_{\max }$ + large $n_{\text {run }}$ ) is also the same region where it has a small contribution to the energy supplied (i.e. low capacity ratio); 
this effect is contrary to what is desired. Analogously, the regions where the High-Power battery observes a high peak power coincide with the areas of the $2 \mathrm{D}$ space where it has a very high capacity ratio and again, this is not desirable because this kind of battery has a high cost per unit energy capacity.

The peak power to energy capacity $(P P t E)$ ratio, calculated via Eq. (11), provides a much clearer view of the aforementioned trade-off. The capacity of either battery (High Capacity and High Power) is proportional to its contribution to the energy requirement in the EPA-LA92 cycle. It should be remembered that the study considers the capacity of the original battery pack in the vehicle $(24 \mathrm{kWh})$ as a constraint and the sum of the capacities of the High-Capacity and High-Power batteries should be equal to it.

$$
P P t E_{H C}=\frac{\left|P_{e a k_{H C}}\right|}{24 * E_{H C}}
$$

Figure 9 shows how the PPtE ratio of both batteries varies with respect to the input parameters of the filter. For the case of the High-Capacity battery, the PPtE ratio needs to be minimized because these batteries have a low cost per unit energy capacity and a high cost per unit power. It can be seen that the region where the ratio is minimized is the region where capacity ratio of the battery $\left(E_{H C}\right)$ oscillates between 0.73 and 0.78 . The absolute minimum value for PPt $E_{H C}$ is 2.67 , which is obtained with a $\omega_{\max }=45$ and a $n_{\text {run }}=9$; here $E_{H C}$ has a value of 0.75 .

On the other hand, the PPtE ratio for the High-Power battery should be maximized because these batteries have a low cost per unit power and a high cost per unit energy capacity. Due to the way the load decomposition is carried out, the region where the PPtE ratio is maximized is the region where the filter has the minimum possible effect $\left(\omega_{\max }=3, n_{\text {run }}=1\right)$. This is a trivial finding since it suggests that the High-Power battery should be as small as possible, to the point where its presence in the system is almost negligible. Figure 9 (bottom) does not show the aforementioned region and focuses only on $\omega_{\max } \geq$ 25 and $n_{\text {run }} \geq 10$. It is interesting to note that (unlike the case for the High-Capacity battery) the PPtE ratio for the HighPower battery decreases continuously with increasing values for $\omega_{\max }$ and number of runs.

Equations (12) and (13) are the assumed cost models for the High-Capacity and High-Power batteries, respectively. It should be emphasized that these simplified models are merely illustrative. Up to now there are no models available in the literature that allow calculating the cost of an electric battery by independently assigning a monetary value to its energy storage capacity and peak power capability.

$$
\begin{aligned}
& \cos _{H C}=20 \cdot\left(24 \cdot E_{H C}\right)+60 \cdot \text { Peak }_{H C} \\
& \cos _{H P}=140 \cdot\left(24 \cdot E_{H P}\right)+30 \cdot \text { Peak }_{H P} \\
& \cos _{\text {total }}=\operatorname{cost}_{H C}+\operatorname{cost}_{H P}
\end{aligned}
$$

The closest to this is a model developed by Sakti et al. [30], which calculates the cost of a NMC Li-ion battery based on experimental data. This work presupposes that the HighPower battery will have a different chemistry to the HighCapacity battery that would allow reducing the cost per unit power. Therefore, Sakti's model could only be applied for the High-Capacity battery. The linear equation proposed for the High-Capacity battery (Eq. (12)) yields very similar costs to Satki et al. model for a battery with specs in the range studied.
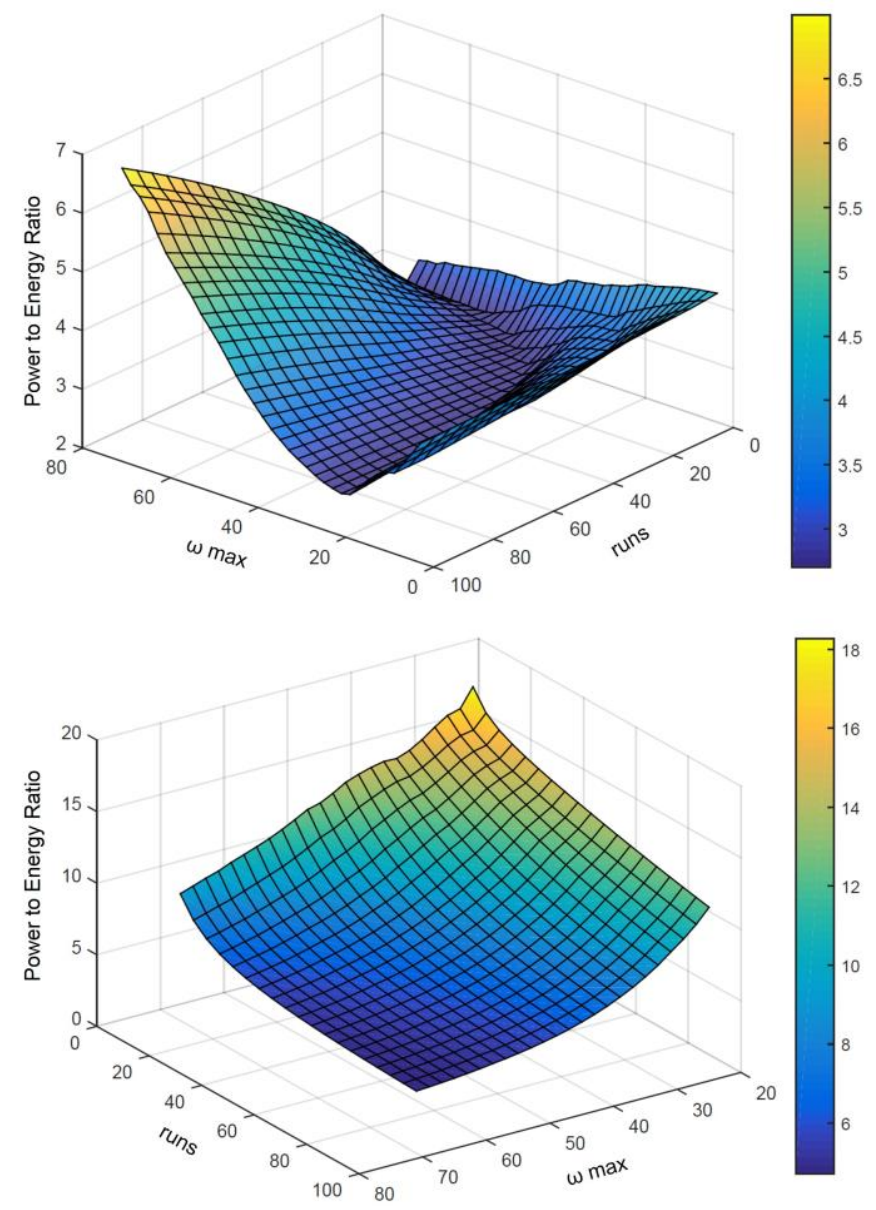

Figure 9. Effect of the filter parameters on the PPtE ratios seen by the High-Capacity (top) and High-Power (bottom) batteries

Using Eq. (12) the cost for the original battery pack of the vehicle ( $24 \mathrm{kWh}$ and $102.35 \mathrm{~kW}$ ) is estimated to be $\$ 6621.1$, which is in agreement with the prices quoted by Nissan for the Leaf's battery pack [31]. It should be noted that the cost of the battery pack not only accounts for the Li-ion cells (192 in the case of the Nissan Leaf) but it also considers the battery management system, sensors, internal wiring, plugs, etc., used to monitor the state of charge of the pack and control some critical parameters such as the temperature of the modules [32].

Figure 10 shows the total cost of the different combinations of batteries (High Capacity + High Power) contained in the twodimensional search space $\left(\omega_{\max }\right.$ and $\left.n_{\text {run }}\right)$. The total cost is calculated, as aforementioned by Eqs. (12)-(14). In the surface, the result of every combination of the filter's control parameters: $n_{\text {run }}$ and $\omega_{\text {max }}$ is shown. 

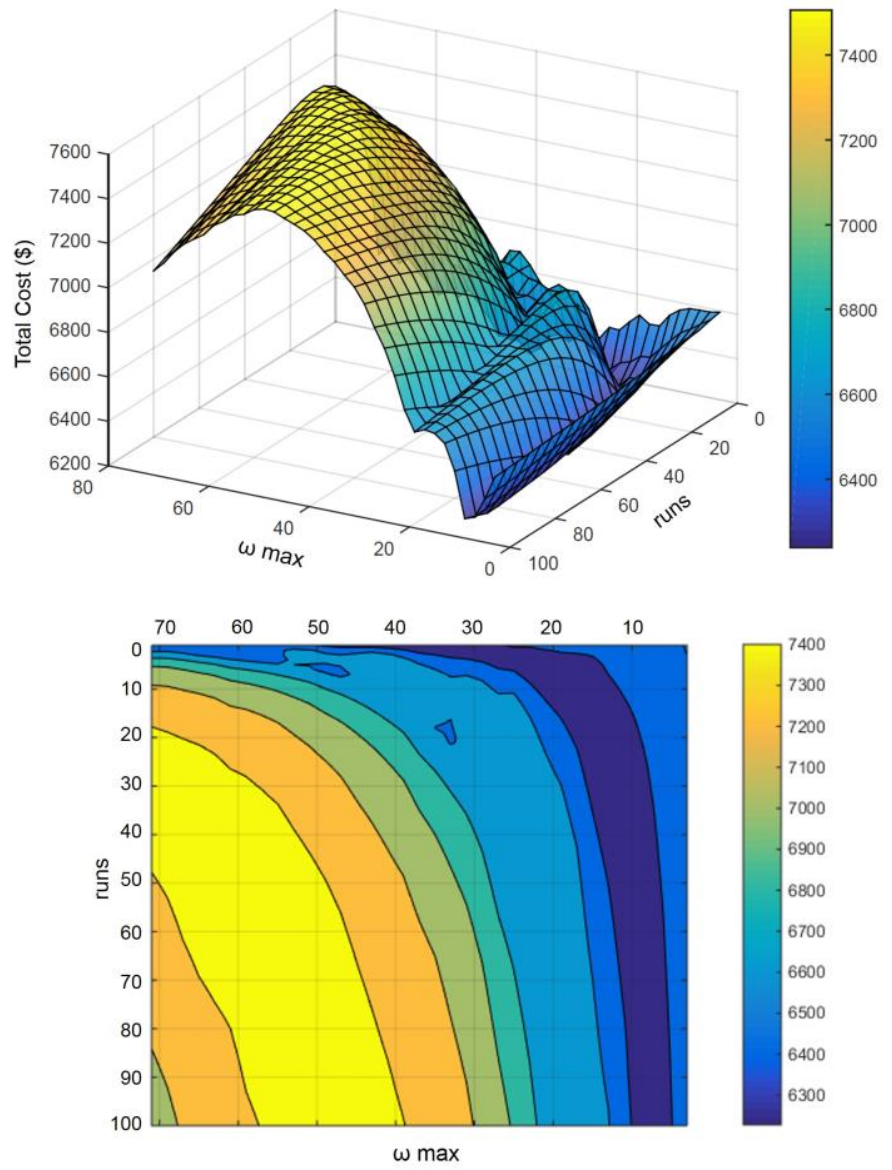

Figure 10. Variation of the total cost of the hybrid battery $(\mathrm{HC}+\mathrm{HP})$ with respect to the filter's control parameters

As earlier explained, each one of those combinations is a different split of the vehicle's load (in this case the EPA-LA92 profile). The High-Capacity and High-Power batteries of every combination presented in the surface has different operating parameters: different capacity ratios (See Fig. 7) and different maximum peak powers (see Fig. 8).

It can be seen in Figure 10 that the total cost surface has local maxima and minima. This behaviour is caused by the shape of the reference signal and the way the filter works, together with the form of Eqs. (12)-(14). The highest overall costs observed are around $\$ 7500$. These are obtained from combinations of $\omega_{\max }$ and $n_{\text {run }}$ that yield an $E_{H C}$ in the range between 0.25 to 0.5

The lowest overall costs, ranging from $\$ 6250$ to $\$ 6350$, are found in the region where the capacity ratio of the HighCapacity battery is between 0.92 and 0.96 . The absolute lowest total cost found is $\$ 6227.8$, which is obtained by means of a $\omega_{\max }=15$ and $n_{\text {run }}=22$. In the optimum configuration found (minimum cost), the High-Capacity battery has a capacity ratio $\left(E_{H C}\right)$ of 0.93 which is equivalent to $22.36 \mathrm{kWh}$ and it sees a maximum peak power of $75.56 \mathrm{~kW}$. Meanwhile, the HighPower battery has a capacity of $1.63 \mathrm{kWh}$ and sees a comparatively large peak power of $33.93 \mathrm{~kW}$
The total cost of $\$ 6227.8$ represents a $5.9 \%$ cost reduction in comparison to the cost of the original battery pack of the vehicle.

Figure 11 provides a different angle at the overall system costs. Here all the 3500 different signal splits (combinations of $\omega_{\max }$ and $n_{\text {run }}$ ) are presented in the form of a Pareto front with respect to the capacity ratio of the High-Capacity battery $\left(E_{H C}\right)$. As it can be seen, many different combinations of parameters yield the same capacity ratio $(+/-0.1 \%)$ but some of them are more expensive than others. The lowest cost of $\$ 6227.8$ is obtained, as aforementioned, with a capacity ratio $\left(E_{H C}\right)$ of 0.93 .

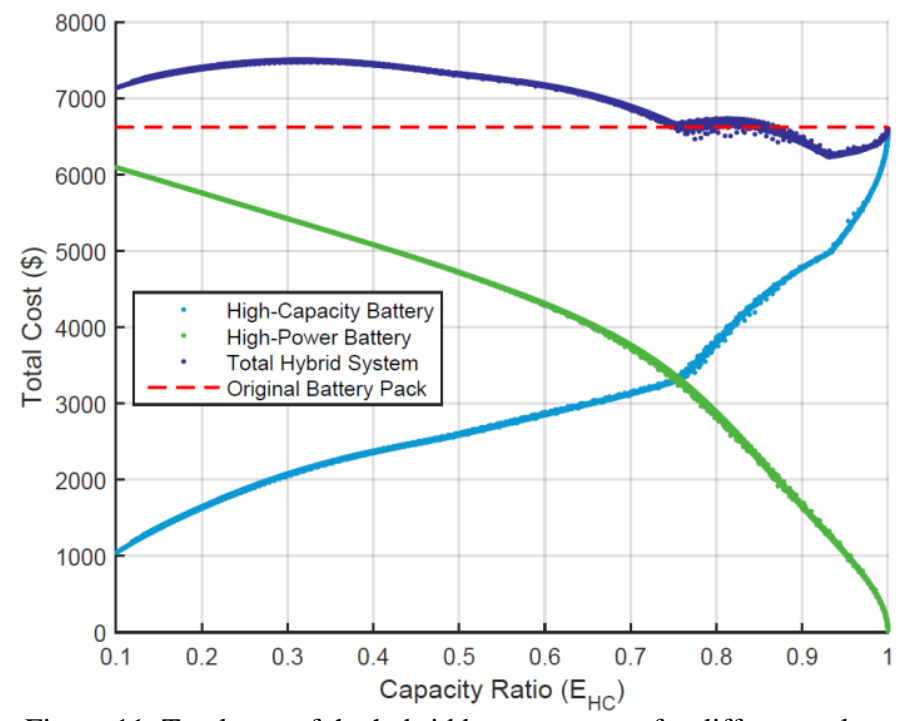

Figure 11. Total cost of the hybrid battery system for different values of capacity ratio $\left(E_{H C}\right)$

It has been demonstrated that the cost of the battery pack can be reduced if the load of the electric motor is split into two frequency components which are handled by two separate batteries: High Capacity (for the low frequency part) and High Power (for the high frequency part).

An important aspect to address regarding the implementation of the hybrid system described (HC+ HP batteries) is the possibility of incurring into additional costs in the rest of the components of the vehicle's powertrain. As Figure 12 shows, the powertrain required for a hybrid battery pack (bottom) will differ slightly from the configuration used in a normal batterypowered EV, such as the Nissan Leaf (top).

In the standard configuration (Fig. 12 top), the battery pack is first connected to a DC/DC converter and a DC Link. In the Nissan Leaf, these two components are integrated into a single unit known as the "DC Junction Box" (part number 292C03NA0C). The main functions of the DC Junction Box are acting as a coupling interphase between the battery pack and the inverter and to supply low-voltage power for the $12 \mathrm{~V}$ electric devices in the car. The output of the DC Junction Box is fed into an inverter which produces $\mathrm{AC}$ to drive the electric motor [33]. Needless to say, all the aforementioned 
components are bidirectional to allow the recharge of the batteries through the regenerative braking system.
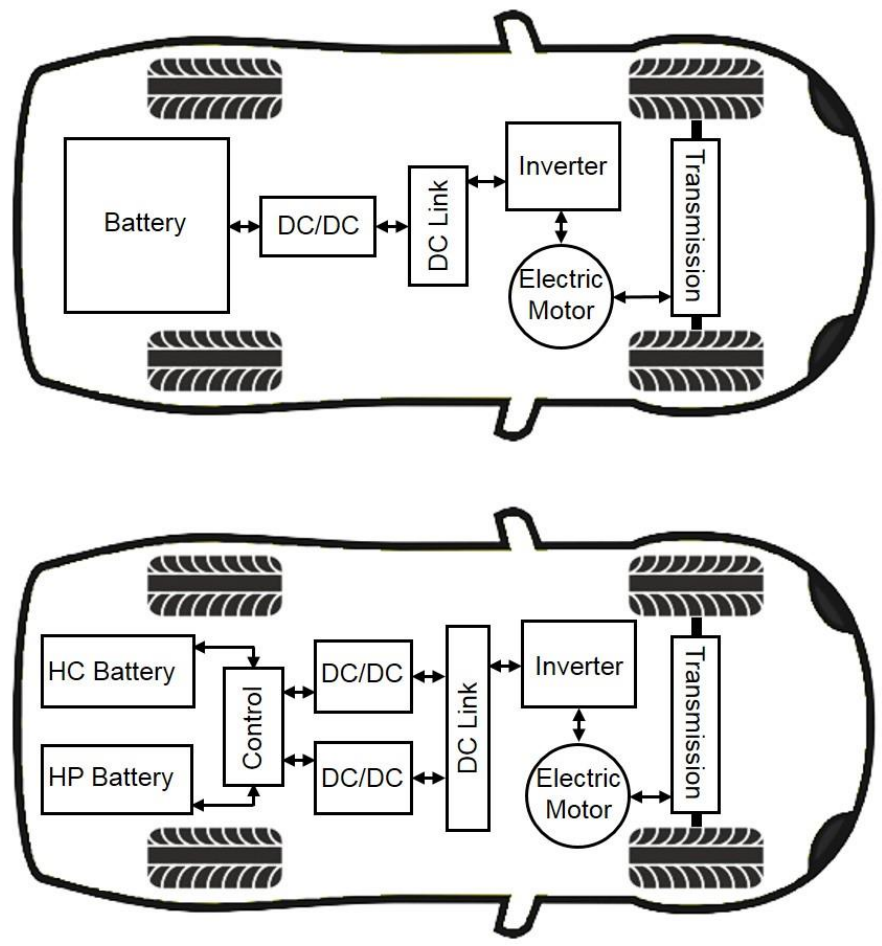

Figure 12. Comparative diagram of the two powertrains: Single battery and hybrid (HC+HP) battery

The inverter, electric motor and transmission remain unchanged in the hybrid-battery configuration (Fig 12 bottom), thus the potential for an increase in cost in these components is discarded. However, the DC/DC converter and DC Link (known collectively as the Junction Box) will be slightly different. In the hybrid-battery configuration two independent DC/DC converters will be required because it is likely that the output voltages of the High Capacity and High Power batteries differ from each other (given that different chemistries will be used). If the output voltages of the two batteries were the same, probably one DC/DC converter (with capability for two simultaneous inputs) would be enough.

The junction box of the Nissan Leaf costs approximately $\$ 650$ $[34,35]$. The cost of the power electronics contained in it is given primarily by their power rating. Even if two junction boxes were required for the proposed hybrid-battery configuration, the overall cost would remain the same, or very similar, because (as explained in section 2.3) the sum of the power supplied by the two batteries at any one time is the same as in the single battery configuration.

In reality the cost of installing two smaller junction boxes will be higher than installing a single larger box due to the cost of the casings, connectors, wiring etc; however the cost of these components is negligible in comparison to the cost of the whole unit. It should also be noted that only one of the two DC/DC converters (linked to the HC battery) will act as a low voltage source for the vehicle's peripheral electronics, while the other one (linked to the HP battery) will be a much simpler circuit.

Because the two batteries will not always operate in parallel, (e.g. when one is active and the other one is idle) a control unit is required. This unit will monitor the load of the electric motor and will identify which battery should supply the power at any given time. The hardware for the control unit will not be considerably different from any other ECU (electronic control unit) found in the vehicle thus its cost should not exceed $\sim$ \$100. Perhaps the main difference is that the control unit will be required to pass high voltage lines $(\sim 360 \mathrm{~V})$ coming from the batteries and to switch them on/off as appropriate.

The software programmed in the control unit will indeed be complex but it will not have a perceivable effect on the cost when these control units are mass produced. The software could be based on a modified version of the sign-preserving filter presented herein. For this it would need to be modified to work on a rolling basis and having only a short forecast (of a few seconds at most) instead of knowing the shape of the full signal. Alternatively, the software could be based on a conventional (albeit advanced) control strategy that takes into account, among other parameters, the state of charge of both batteries and the change in the motor's load in order to predict if there is a peak in power demand coming.

It results credible, based on the above, that the hybrid 2-battery system proposed can be implemented in an EV with only a marginal increase in the cost of the other components of the vehicle's powertrain. Therefore, the reduction achieved in the cost of the battery pack will be reflected in the overall cost of the vehicle.

Besides the reduction in the capital cost of the battery pack, the proposed signal-splitting methodology offers a secondary benefit. Because the hybrid battery consists in two separate batteries, one tailored for bulk energy storage and another one tailored for peak power, the life expectancy of both is increased in comparison with the normal single battery approach.

In the single battery approach, the chemistry of the battery is tuned to achieve a middle-point between specific power and specific energy. Because of this, the battery sustains damage and its lifespan is reduced when it is required to supply power peaks. In the 2-battery approach, the power peaks are handled by the HP battery. This battery has been purposely designed for high PPtE ratios, so it will not degrade when subjected to intense discharges. The HC battery supplies a much steadier load and sees a considerably lower PPtE ratio, thus its lifespan is not shortened.

The prolonged (or better preserved) life expectancy of the HC and HP batteries improves the cost of running the EV vehicle since they won't need to be replaced so often. For reference, in a Nissan Leaf the battery lasts on average 5 years (or 60,000 miles) [31]. An assessment of the improvement in battery life is out of the scope of this paper given that the models available in the literature (e.g.[36]) are chemistry-specific. It is 
impossible to quantify the improvement in lifespan achieved by means of the proposed hybrid system without defining the specific chemistries of both, the HC and HP batteries.

\subsection{Case study 2: Modified profile based on EPA-LA92}

In section 3.1 the optimization of the battery pack for a Nissan Leaf was carried out using as a reference load the electric power profile of the car when subjected to the EPA-LA92 driving cycle. The results presented are positive and show that a reduction in the total cost of the battery of about $5.9 \%$ can be achieved.

Although a meaningful percentage in cost reduction was achieved, the number is limited by the power profile used for the analysis and does not fully demonstrate the potential of the sign-preserving filter. The filter would certainly be more effective if the signal to be split had a larger crest factor (i.e. a peakier profile). This section of the paper presents a second case study in which a signal with a higher crest factor is used with aims at further demonstrating the capabilities of the signpreserving filter as a tool for optimizing the battery pack of an EV.

Instead of using an entirely arbitrary signal with a higher crest factor, a structured approach to define the signal used for the second case study was followed. The signal for the case study no. 2 is created by modifying the electric power profile used in the previous case study by means of Eqs.(15)-(17), where $x$ are the data-points from the EPA-LA92 power profile (in $\mathrm{kW}$ ) and $f(x)$ is their equivalent value in the modified profile. For example, at any time $(t)$ when the original power profile has a value of $40 \mathrm{~kW}$, the modified signal will have a value of 23.88 $\mathrm{kW}$ and at any time $(t)$ when the original power profile has a value of $100 \mathrm{~kW}$, the modified signal will have a value of $102.9 \mathrm{~kW}$

$$
f(x)=\left\{\begin{array}{cc}
\frac{1}{2.5} \cdot(M+L) & x>0 \\
0 & x=0 \\
-\frac{1}{2.5} \cdot(M+L) & x<0
\end{array}\right.
$$

Where:

$$
\begin{aligned}
& M=300 * \exp \left(-\left(\frac{|x|-130}{50}\right)^{2}\right) \\
& L=100 * \exp \left(-\left(\frac{|x|-70}{35}\right)^{2}\right)
\end{aligned}
$$

The form of Eqs. (15)-(17) was purposely chosen to perform a "selective squashing function" on the original signal. The points that are closer to zero are scaled down by a larger factor than those points further away from zero; thus the most prominent peaks of the original signal remain almost unmodified. This allows reducing the $R M S$ value while maintaining a large peak value, which yields an increased crest factor.
The objectives of following the procedure above-described over simply utilizing an arbitrary signal are:

1) If the signal generated is converted back to a speed profile applying the VT-CPEM model backwards it will still resemble a driving pattern, albeit a more aggressive one.

2) It enables the results presented in the paper to be reproduced more easily. The reader can download the full EPA-LA92 driving profile (1435 data points) from [15] and generate the same signal via Eqs. (15)-(17).

Figure 13 shows the modified power profile, hereafter referred to as "Mod-EPA". It can be seen in the figure that the ModEPA profile appears to be peakier, which is confirmed by its increased crest factor of 11.49 (in contrast to the 6.3 of the original EPA-LA92).

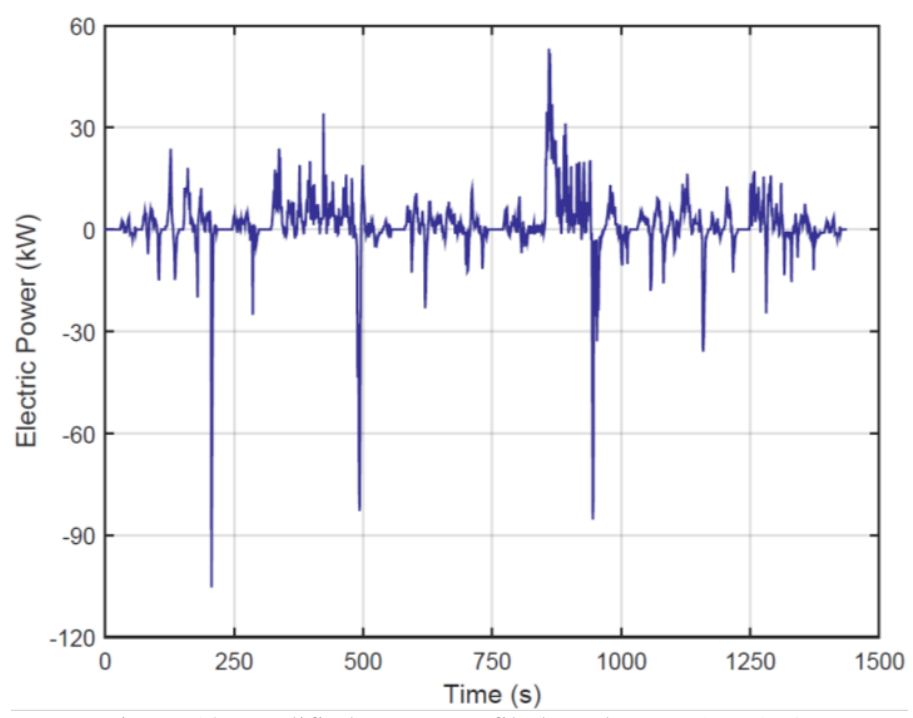

Figure 13. Modified power profile based on EPA_LA92

The Mod-EPA profile has a peak power of $105.4 \mathrm{~kW}$ and an average (RMS) power of $9.17 \mathrm{~kW}$. At the end of the "driving cycle" the net energy outflow from the battery is $0.54 \mathrm{kWh}$; throughout the cycle the battery supplies $1.15 \mathrm{kWh}$ to the electric motor but receives back $0.61 \mathrm{kWh}$ from the regenerative brakes of the car.

The cost-optimization is carried out in the same way as in the case study no.1. A two-dimensional search space is created with different values for $\omega_{\max }$ and $n_{\text {run }}$. The maximum wave width in this case goes from 3 to 45 points (excluding even values) while the number of runs goes from 1 up to 100 , which results in 2200 different combinations of parameters.

Figure 14 shows the capacity ratio of the High-Capacity battery obtained for different combinations of filter parameters. A very similar behaviour to that observed in the previous case study can be seen. For small values of $\omega_{\max }$ and $n_{\text {run }}$ the capacity ratio of the High-Capacity battery is very high, while it decreases with increasing values for the maximum wavewidth and number of runs. 


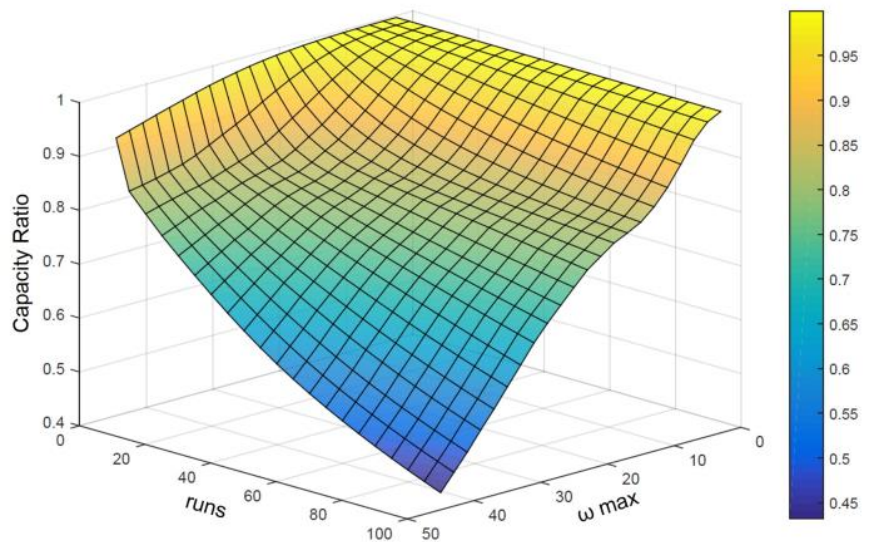

Figure 14. Behaviour of the capacity ratio of the High-Capacity battery for different splits $\left(\omega_{\max }+n_{\text {run }}\right)$ of the Mod-EPA profile

The variation of the peak power observed by both batteries High-Capacity and High-Power, as the filter parameters change is shown by Figure 15. Similarly to case study no. 1, at low values of $\omega_{\max }$ and $n_{\text {run }}$ the peak power seen by the HighCapacity battery approximates the peak power of the unfiltered Mod-EPA profile (105.4 kW) and the peak power seen by the High-Power battery is very low. Inversely, at high values of $\omega_{\max }$ and $n_{\text {run }}$ the peak power values seen by the HighCapacity battery markedly reduce whilst the peak powers experienced by the High-Power battery approximate the absolute maximum peak power of the unfiltered Mod-EPA signal.
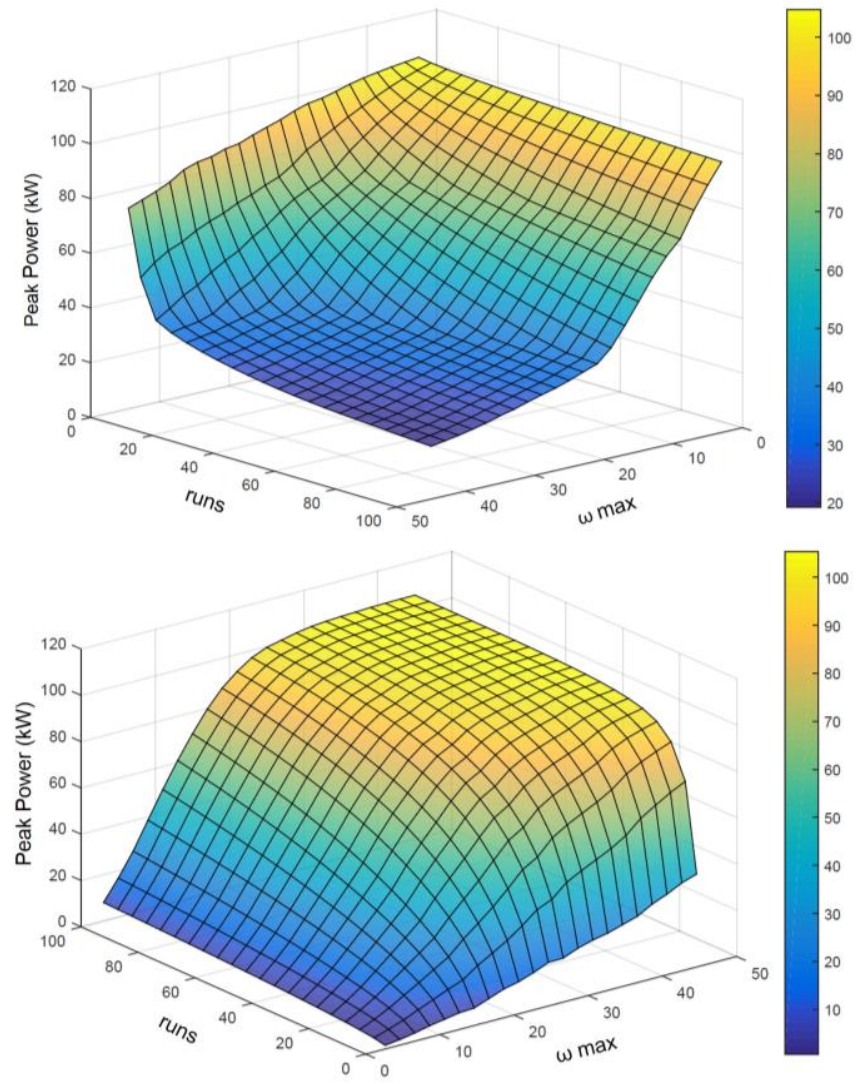

Figure 15. Behaviour of the peak power seen by the High-Capacity (top) and High-Power (bottom) batteries for different splits of the Mod-EPA profile
Combining the results shown in Figures 14 and 15 it is possible to calculate a Peak Power to Energy Capacity (PPtE) ratio for both batteries by means of Eq. (11). This ratio is useful because it reveals the combinations of parameters that simultaneously maximize the energy contribution and minimize the power seen by the High-Capacity battery or the inverse case for the High-Power battery.

Figure 16 shows the behaviour of the PPtE ratios of both batteries with respect to the filter's control parameters. For the case of the High-Capacity battery it can be seen that the region where the ratio is minimized is the region where the capacity ratio of the battery $\left(E_{H C}\right)$ neighbours $0.55-0.65$. The absolute minimum value for PPtE is 1.69 , which is obtained with a $\omega_{\max }=41$ and a $n_{\text {run }}=56$; here $E_{H C}$ has a value of 0.61 .

Similar to the case study no.1, the region where the PPtE ratio for the High-Power battery is maximized is the region where the minimum $E_{H P}$ is found $\left(\omega_{\max }=3, n_{\text {run }}=1\right)$. The figure does not show this region of the combinatorial space as it is a trivial finding and focuses only on $\omega_{\max } \geq 15$ and $n_{\text {run }} \geq 10$. The PPtE of the High-Power battery decreases as the $\omega_{\max }$ and number of runs increase. It is interesting to note that the slope of the surface is much more pronounced at the corners of the search space, that is small $\omega_{\max }$ and small $n_{\text {run }}$ or for large values of both variables.
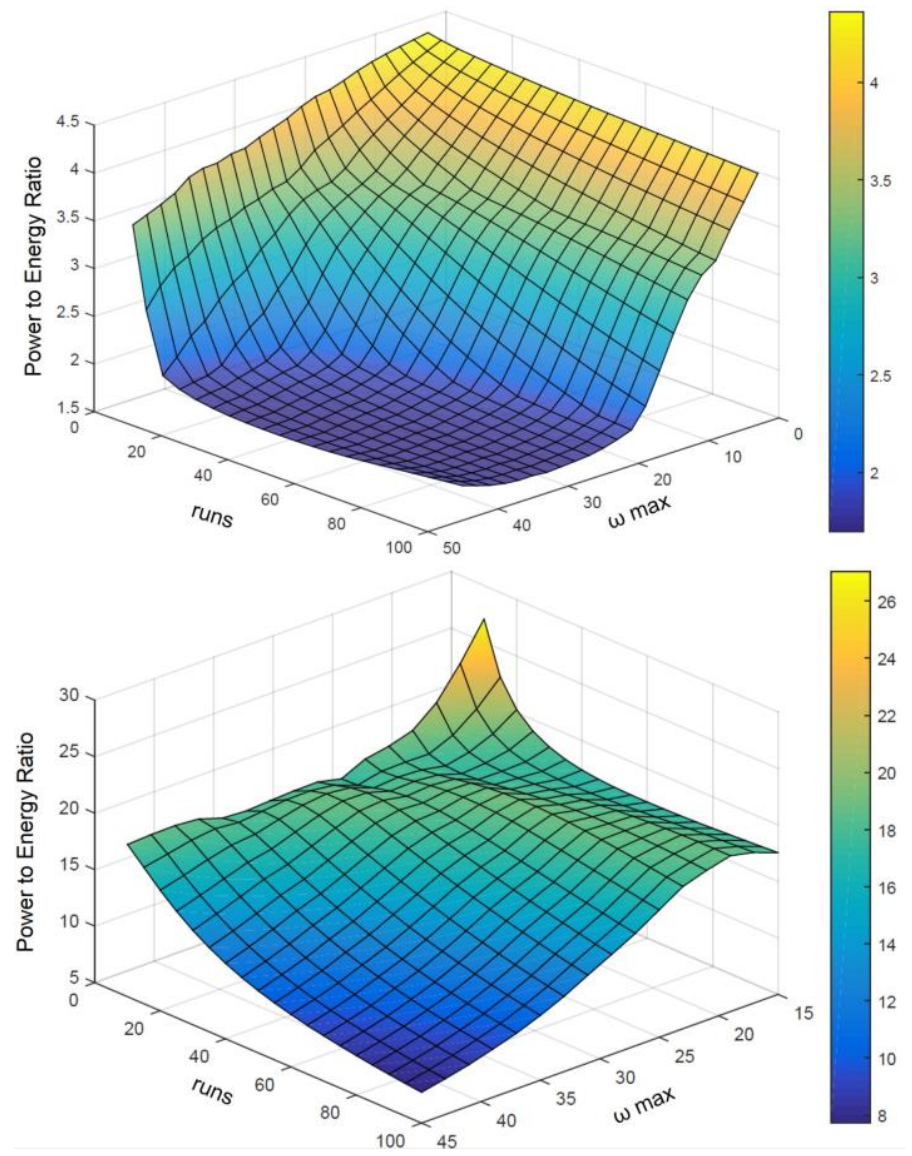

Figure 16. Behaviour of the PPtE ratio of the High-Capacity (top) and High-Power (bottom) batteries for different splits of the Mod-EPA profile 
The cost model described in the previous section (3.1) is used for this case study as well. The cost of the original battery is estimated at $\$ 6804.5(24 \mathrm{kWh}+105.4 \mathrm{~kW})$. The total system cost for each of the 2200 combinations of High-Capacity and High-Power batteries is calculated by means of Eq.(12)-(14) using as inputs the results for capacity ratio (see Fig. 14) and peak power (see Fig.15) obtained from the exploration of the two-dimensional search space $\left(\omega_{\max }\right.$ and $\left.n_{\text {run }}\right)$

Figure 17 shows how the total system cost varies with respect to the two signal-splitting parameters $\left(\omega_{\max }\right.$ and $\left.n_{\text {run }}\right)$. The highest overall costs observed range between $\$ 6700$ and $\$ 6800$. These costs are found in the region formed by a $\omega_{\max } \leq$ 15 and a $n_{\text {run }} \leq 10$. In this region the capacity ratio $E_{H C}$ is higher than 0.97; in other words, the High-Capacity battery provides $97 \%$ or more of the total energy required by the electric motor.
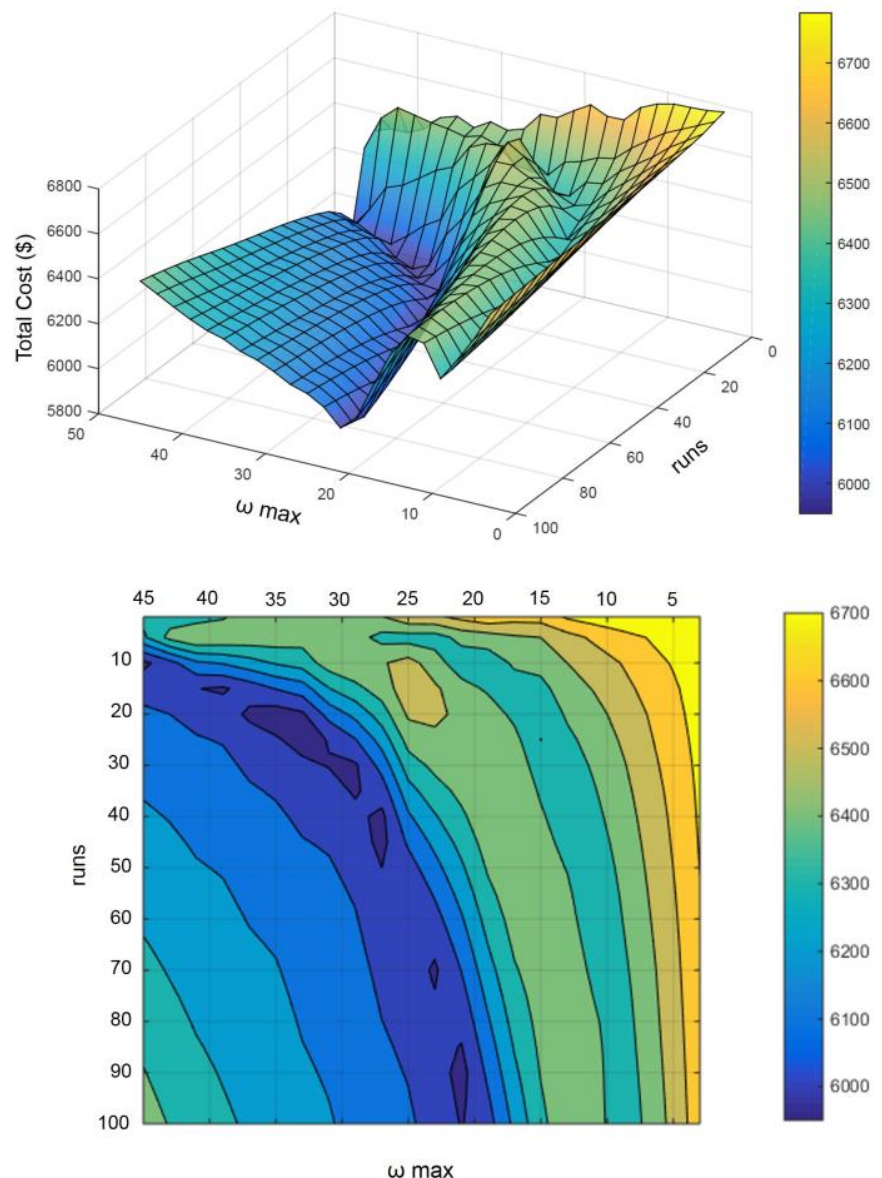

Figure 17. Total cost of the hybrid battery $(\mathrm{HC}+\mathrm{HP})$ obtained for different splits of the Mod-EPA profile

It can be seen that the search-space contains a region where a local minimum is found. In this region the capacity ratio is in the range of $0.9 \leq E_{H C} \leq 0.93$ and system costs around $\$ 6350$ are attained. This feature of the total-cost surface is caused by the combination of diverse factors: 1) the shape of the reference signal being used (Mod-EPA), 2) the mechanics of the operation of the sign-preserving filter and 3) the cost model employed.
A low-cost area (the objective of the optimization) with costs between $\$ 5950$ and $\$ 6050$ is found in the region where the capacity ratio $E_{H C}$ is in a range between $0.78-0.82$. The absolute minimum total cost of $\$ 5939.3$ is obtained with a combination of $\omega_{\max }=33$ and a $n_{\text {run }}=22$. In the optimum configuration found (minimum cost) the High-Capacity battery has a capacity ratio $\left(E_{H C}\right)$ of 0.80 which is equivalent to 19.23 $\mathrm{kWh}$ and it sees a maximum peak power of $37.18 \mathrm{~kW}$. On the other hand, the High-Power battery has a capacity of $7.77 \mathrm{kWh}$ and sees a much larger peak power of $88.55 \mathrm{~kW}$

Figure 18 shows how the overall system cost varies with respect to the capacity ratio of the High-Capacity battery $\left(E_{H C}\right)$. The fact that there are many different combinations of filter parameters $\left(\omega_{\max }\right.$ and $\left.n_{\text {run }}\right)$ that yield the same capacity ratio becomes evident in the figure. It is also clear that for the same capacity ratio some of those combinations are more expensive than others.

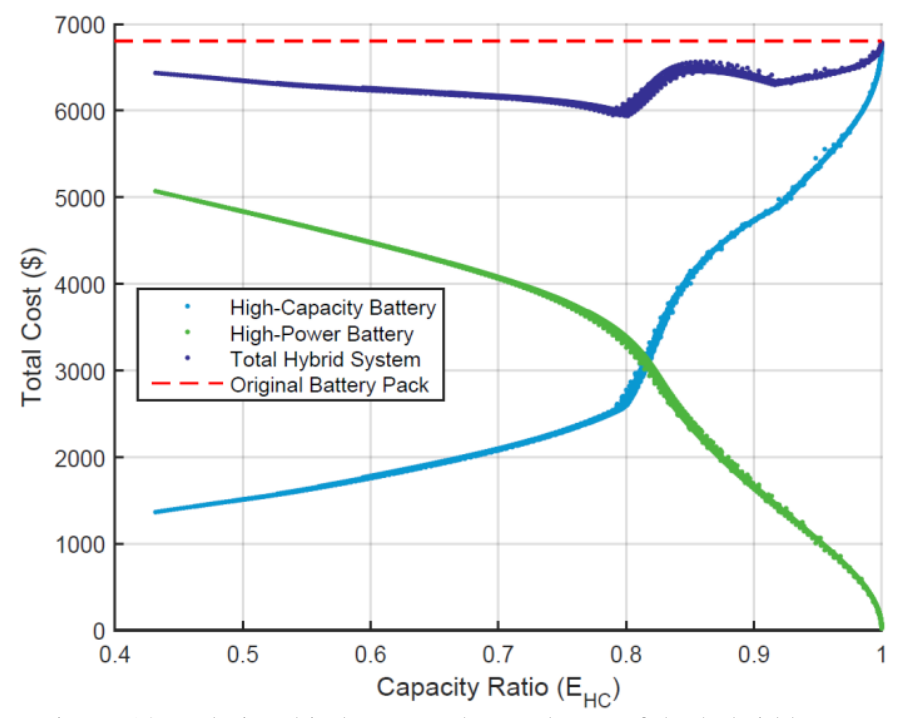

Figure 18. Relationship between the total cost of the hybrid battery and the capacity ratio of the High-Capacity battery $\left(E_{H C}\right)$

A point that is worth noting is that in this case study (considering the Mod-EPA profile) there are no combinations of filter parameters (within the space studied) that produce a higher system cost than that of the reference battery. In other words, regardless of how the hybrid battery system is embodied a reduction in cost will be achieved.

It can be observed in Figure 18 that, as aforementioned, a local minimum of $\$ 6298$ exists at a $E_{H C}$ of 0.92 , while the global minimum $\$ 5939.3$ is located at a $E_{H C}$ of 0.80 . The optimum value found is equivalent to a $12.7 \%$ reduction in cost in comparison to the cost of the original battery pack of the vehicle.

\section{Conclusions}

A study on the cost-optimization of the battery-pack of an EV has been undertaken and is presented in this paper. The study has two main objectives: (i) demonstrate the use of a "SignPreserving" signal processing tool in an EV context and (ii) 
explore the possibility of reducing the cost of an EV's battery pack by replacing it with a hybrid 2-battery system, in which one battery (low frequency) is used for bulk energy storage and the other battery (high frequency) is used for supplying power peaks.

Two case studies were carried out using the Nissan Leaf (which has a $24 \mathrm{kWh}$ battery) as the reference vehicle. The optimization methodology is not limited to passenger cars but could be applied to electric bikes, city buses, etc., being particularly effective for vehicles that experience frequent of drastic acceleration/braking.

The first case study is based on the EPA-LA92 driving cycle. The optimum design for the hybrid system found has a total cost of \$6227.8.In this configuration the High-Capacity battery contributes $93.18 \%$ of the $24 \mathrm{kWh}$ total energy storage capacity and sees a maximum peak power of $75.56 \mathrm{~kW}$. On the other hand, the High-Power battery has a capacity of $1.63 \mathrm{kWh}$ (6.8\% of the total system capacity) and experiences a comparatively large peak power of $33.93 \mathrm{~kW}$. The HighCapacity battery accounts for $79.9 \%$ of the cost of the hybrid battery pack whilst the rest is attributable to the High-Power counterpart. The total cost of $\$ 6227.8$ attained is equivalent to a $5.9 \%$ cost reduction in comparison to the cost of the original battery pack of the vehicle.

The second case study uses a modified profile based on the EPA-LA92 but with an $80 \%$ larger crest factor. The optimum design found attained a cost as low as \$5939.3. In this configuration the High-Capacity battery has an energy storage capacity of $19.23 \mathrm{kWh}$, which is $\sim 80.14 \%$ of the total capacity of the vehicle and sees a maximum peak power of $37.18 \mathrm{~kW}$. The High-Power battery has a smaller capacity of $7.77 \mathrm{kWh}$ but handles a much larger maximum peak power of $88.55 \mathrm{~kW}$. The total cost of the hybrid battery pack is split in a $44-56 \%$ proportion between the High-Capacity and High-Power batteries, respectively. The total cost of $\$ 5939.3$ achieved by the optimization represents a $12.7 \%$ reduction in cost with respect to the cost of the original $24 \mathrm{kWh}$ battery pack of the vehicle.

The study has demonstrated the usefulness of the "SignPreserving filter" in the context of electric vehicles, where the two frequency components need to have the same sign at all times (i.e. no counter-flow of energy). By replacing the battery pack of the vehicle with a hybrid system comprising two batteries (one for bulk energy storage and one for peak-power) cost reductions of $12.7 \%$, or more depending on the load used as a reference, can be achieved.

\section{Acknowledgements}

This research work has been funded by the UK Engineering and Physical Sciences Research Council through the NextGen-TEST and Manifest (EP/N032888/1) projects.

\section{References}

[1] J. Dulac. (May 2013) Global transport outlook to 2050: Targets and scenarios for a low-carbon transport sector.
Presented at the "Mobility: Technology Priorities and Strategic Urban Planning" Workshop in Espoo, Finland.

[2] International Energy Agency (2012). Chapter 13: Transport. In Energy Technology Perspectives 2012 (pp.423455). Paris, France.

[3] U.S. Energy Information Administration. Monthly Energy Review. Table 2.1 Energy consumption by sector. Available online from: https://www.eia.gov/totalenergy/data/monthly/pdf/sec2_3.pdf [4] US. Energy Information Administration. Chapter 8: Transportation sector energy consumption. In International Energy Outlook 2016 (pp. 127 - 137). Washington DC, USA.

[5] C. Gomes. (January 2018) Global Auto Report: Ninth Consecutive Global Sales Record Expected in 2018. Retrieved from:

http://www.gbm.scotiabank.com/scpt/gbm/scotiaeconomics63/ GAR_2018-01-30.pdf

[6] N.Winton (December 2017) Global Car Sales To Gather Momentum In 2018. Forbes. Retrieved from: https:/www.forbes.com/sites/neilwinton/2017/12/28/globalcar-sales-to-gather-momentum-in-2018-while-new-technologydisruption-lurks/\#641cf0ba402b

[7] US. Energy Information Administration. Chapter 9: Energy-related $\mathrm{CO} 2$ emissions. In International Energy Outlook 2016 (pp. 139 - 148). Washington DC, USA.

[8] R. Sims, R. Schaeffer, F. Creutzig, X. Cruz, M. D’Agosto, D. Dimitriu, M.J. Figueroa, L. Fulton, S. Kobayashi, O. Lah, A. McKinnon, P. Newman, M. Ouyang, J. J. Schauer, D. Sperling, G. Tiwari. (2014) Chapter 8: Transport. In: Climate Change 2014: Mitigation of Climate Change. Eds. O. Edenhofer, R. Pichs-Madruga, Y. Sokona. Cambridge University Press, New York, USA.

[9] X. Wu, D. Freese, A. Cabrera, W.A. Kitch. Electric vehicles' energy consumption measurement and estimation. Transportation Research Part D. 2015; 34: 52-67.

[10] International Energy Agency (2017) EV Deployment. In Global EV Outlook 2017: Two million and counting (pp.1228). Paris, France.

[11] OECD/ITF (2017) Chapter 2: Transport demand and Co2 emissions to 2050. In ITF Transport Outlook 2017(pp.47-65). OECD Publishing, Paris, France.

[12] T.J. Barlow, S.Latham, I.S.McCrae, P.G.Boulter. A reference book of driving cycles for use in the measurement of road vehicle emissions". TRL Limited, June 2009.

[13] M. Knowles, H. Scott, D. Baglee. The effect of driving style on electric vehicle performance, economy and perception. International Journal of Electric and Hybrid Vehicles. 2012; 4: 228-47.

[14] M. DeGennaro, E. Paffumi, H. Scholz, G. Martini. GISdriven analysis of e-mobility in urban areas: An evaluation of the impact on the electric energy grid. Applied Energy. 2014;124:94-116.

[15] US Environmental Protection Agency. Dynamometer Drive Schedules. Available online at: https://www.epa.gov/vehicle-and-fuel-emissionstesting/dynamometer-drive-schedules

[16] C. Fiori, K. Ahn, H. Rakha. Power-based electric vehicle consumption model: Model development and validation. Applied Energy. 2016; 168:257-268. 
[17] S. Shibata, T. Nakagawa. Mathematical model of electric vehicle power consumption for traveling and air-conditioning. Journal of Energy and Power Engineering. 2015; 9:269-275.

[18] R. Abousleiman, O. Rawashdeh. Energy consumption model of an electric vehicle. In: Transportation electrification conference and expo (ITEC), 2015 IEEE. IEEE; 2015

[19] J.G. Hayes, K. Davis. Simplified electric vehicle powertrain model for range and energy consumption based on EPA coast-down parameters and test validation by Argonne National Lab data on the Nissan Leaf. In: Transportation electrification conference and expo (ITEC), 2014 IEEE. IEEE; 2014

[20] M. De Gennaro, E. Paffum, G. Martini, U. Manfredi, S. Vianelli, F. Ortenzi, A. Genovese. Experimental test campaign on a battery electric vehicle: laboratory test results (Part 1). Int. Journal of Alternative Powertrains 2015;4:100-14.

[21]US. Department of Energy. Advanced vehicle testing activity (AVTA) of the Idaho Nation Laboratory (INL). Retrieved from: https://avt.inl.gov/vehicle-button/2011-nissanleaf

[22] R. Maia, M. Silva, R. Araujo, U. Nunes. Electrical vehicle modelling: a fuzzy logic model for regenerative braking. Expert systems with applications. 2015; 42:8504-8519.

[23] H. Rakha, I. Lucic, S.H. Demarchi, J.R. Setti, M. Van Aerde. Vehicle dynamics model for predicting maximum truck acceleration levels. Journal of Transportation Engineering. 2001; 127: 418-425.

[24] H.A Rakha, K. Ahn, K. Moran, B. Saerens, E. Van den Bulck. Virginia tech comprehensive power-based fuel consumption model: model development and testing. Transport Res Part D: Transp Environ 2011; 16:492-503.

[25] B. Cárdenas, T.R. Davenne, S.D. Garvey. A Signpreserving filter for signal decomposition. Journal of Systems and Control Engineering.2018. In Press.

[26] R. Yakushi. (May 2012) EV/HEV Safety. Presented at the Electric Vehicle Safety Technical Symposium of the NHTSA. Washington DC. Available online at :

https://one.nhtsa.gov/Vehicle-Safety/Electric-Vehicle-Safety-

Symposium

[27] The Battery University (2018), "Types of Lithium-ion" Available online at:

http://batteryuniversity.com/learn/article/types of lithium ion [28] G. Zubi, R. Dufo-Lopez, M. Carvalho, G. Pasaoglu. The lithium-ion battery: State of the art and future perspectives. Renewable and Sustainable Energy Reviews, 2018; 89:292308.

[29] A.M. Andwari, A. Pesiridis, S. Rajoo, R. Martinez-Botas, V. Esfahanian. A review of battery electric vehicle technology and readiness levels. Renewable and Sustainable Energy Reviews, 2017; 78:414-430.

[30] A. Satki, J.J. Michalek, E.R.H. Fuchs, J.F. Whitacre. A techno-economic analysis and optimization of Li-ion batteries for light-duty passenger vehicle electrification. Journal of Power Sources, 2015; 273:966-980.

[31] J. Burn (8 December 2014) Nissan leaf battery replacement to cost $£ 4920$. Auto Express UK. Retrieved from: http://www.autoexpress.co.uk/nissan/89694/nissan-leafbattery-replacement-to-cost-4920
[32] Automotive Energy Supply Corporation (2018). Cell, module and pack for EV applications. Available online at: http://www.eco-aesc-lb.com/en/product/liion_ev/

[33] Automotive Industry Portal Mark-Lines (September 2012) Nissan Leaf teardown (Part 2): main components disassembled. Available online at:

https://www.marklines.com/en/report_all/rep1104_201209\#rep ort_area_3

[34]Factory Nissan Parts (2018) Available online at: https://www.factorynissanparts.net/oem-parts/nissan-junctionboard-292c03na0c

[35] H. Bängtsson, M. Alaküla. Swedish Electric and Hybrid Vehicle Centre (June 2015). Cost Analysis of Electric Land Transport

[36]J. Wang, P. Liu, J. Hicks-Garner, E. Sherman, S. Soukiazian, M. Verbrugge, H. Tataria, J. Musser, P. Finamore. Cycle-life model for graphite $\mathrm{LiFePO} 4$ cells. Journal of Power Sources, 2011; 196:3942-3948. 